\title{
Benchmarking UMI-based single-cell RNA-seq preprocessing workflows
}

\author{
Yue You ${ }^{1,2^{*}}$, Luyi Tian ${ }^{1,2}$, Shian Su ${ }^{1,2}$, Xueyi Dong ${ }^{1,2}$, Jafar S. Jabbari ${ }^{3,4}$, Peter F. Hickey ${ }^{1,2,5^{*}}$
} and Matthew E. Ritchie ${ }^{1,2,6^{*}}$ (I)

*Correspondence:
you.y@wehi.edu.au;
hickey@wehi.edu.au;
mritchie@wehi.edu.au
'Epigenetics and Development
Division, The Walter and Eliza Hall
Institute of Medical Research, 1G
Royal Parade, Parkville, Australia
2Department of Medical Biology,
The University of Melbourne,
Parkville, Australia
Full list of author information is
available at the end of the article

(c) The Author(s). 2021 Open Access This article is licensed under a Creative Commons Attribution 4.0 International License which permits use, sharing, adaptation, distribution and reproduction in any medium or format, as long as you give appropriate credit to the original author(s) and the source, provide a link to the Creative Commons licence, and indicate if changes were made. The images or other third party material in this article are included in the article's Creative Commons licence, unless indicated otherwise in a credit line to the material. If material is not included in the article's Creative Commons licence and your intended use is not permitted by statutory regulation or exceeds the permitted use, you will need to obtain permission directly from the copyright holder. To view a copy of this licence, visit http://creativecommons.org/licenses/by/4.0/. The Creative Commons Public Domain Dedication waiver (http://creativecommons.org/publicdomain/zero/1.0/) applies to the data made

available in this article, unless otherwise stated in a credit line to the data.

\begin{abstract}
Background: Single-cell RNA-sequencing (scRNA-seq) technologies and associated analysis methods have rapidly developed in recent years. This includes preprocessing methods, which assign sequencing reads to genes to create count matrices for developed to provide users with convenient tools for handling this process, how they compare to one another and how they influence downstream analysis have not been
\end{abstract}

Results: Here, we systematically benchmark the performance of 10 end-to-end preprocessing workflows (Cell Ranger, Optimus, salmon alevin, alevin-fry, kallisto bustools, dropSeqPipe, scPipe, zUMIs, celseq2, and scruff) using datasets yielding different biological complexity levels generated by CEL-Seq2 and 10x Chromium platforms. We compare these workflows in terms of their quantification properties directly and their impact on normalization and clustering by evaluating the performance of different method combinations. While the scRNA-seq preprocessing workflows compared vary in their detection and quantification of genes across datasets, after downstream analysis with performant normalization and clustering methods, almost all combinations produce clustering results that agree well with the known cell type labels that provided the ground truth in our analysis.

Conclusions: In summary, the choice of preprocessing method was found to be less important than other steps in the scRNA-seq analysis process. Our study comprehensively compares common scRNA-seq preprocessing workflows and summarizes their characteristics to guide workflow users.

Keywords: scRNA-seq, Transcriptomics, Methods comparison, Sequencing analysis, Preprocessing

\section{Background}

Over the past decade, single-cell RNA-sequencing (scRNA-seq) technologies and associated analysis methods have rapidly developed and been applied to a wide range of biological systems [1, 2]. The large number of analysis methods available presents a significant challenge for data analysts who are left to choose which of the many tools are 
best suited to their experiment and analysis goals. Fortunately, there are now many systematic benchmarking studies that explore this question in detail [3-6]. However, these evaluations almost exclusively focus on downstream analysis tasks, including normalization, clustering, trajectory analysis, cell type identification, and data integration. They ignore the crucial first step of preprocessing that summarizes the sequencing reads into a count matrix which is used as input to all downstream analyses.

The main difference between preprocessing scRNA-seq data compared with bulk RNAseq lies in having to deal with various DNA barcodes which assist in the assignment of sequence reads to their cell or molecule of origin $[7,8]$. Most methods are designed to work with unique molecular identifier (UMI) $[9,10]$ based data since these protocols are widely used in the field. UMIs are random oligonucleotide barcodes of a fixed length that are used to distinguish between the original molecules present in the cell and the PCR amplified copies generated during library construction. The process of UMI deduplication is a key part of transcript quantification in scRNA-seq data analysis that aims to provide molecule counts for the expressed genes in each cell and eliminate PCR-related quantification biases.

Typical scRNA-seq preprocessing workflows involve demultiplexing, mapping, transcript quantification, and quality control. An overview of the main steps involved in preprocessing is summarized in Fig. 1 (A). Starting from raw FASTQ files, cell barcodes (CBs) and UMIs are first appended as tags to the header of each cDNA read. Next, cDNAs are aligned to either a reference genome or mapped using a lightweight method to the transcriptome depending on the particular pipeline. To overcome amplification bias, UMIs are collapsed to remove PCR-duplicated molecules from the gene counts in each cell. Base errors in CBs and UMIs are usually corrected at this step or before alignment. Next, reads are separated by CBs and assigned to genes or transcripts which allows the construction of a cell-by-gene count matrix (with cells in the columns and genes/transcripts in the rows). Next, cells of low quality and genes with low abundance are typically filtered out and the resulting count matrix is used in downstream analysis. Of note, CBs are known in advance for each well in plate-based protocols such as CEL-Seq [11] and randomly assigned to cells in droplet-based protocols, like 10x Chromium [12] and inDrops [13]. Because of this, different strategies are applied to construct CB "allow lists," and additional steps to distinguish real cells from ambient RNA are suggested for droplet-based protocols [14].

Researchers can build their own preprocessing workflows by combining individual methods that address each of the aforementioned steps, or they may choose one of the many packaged workflows that have been proposed and published that aim to make this process more convenient. Examples of preprocessing workflows include Cell Ranger [12], UMI-tools [15], scPipe [16], and zUMIs [17]. More recently, alignment-free tools such as kallisto [18] and salmon [19] have been adapted to handle single-cell data to improve the computational efficiency of scRNA-seq data analysis. In addition, projects like the Human Cell Atlas [20] have developed their own preprocessing workflow (Optimus [21]) that uniformly processes the millions of human single-cell transcriptomes generated through this international collaboration. Other examples include the Single Cell Expression Atlas that has applied the SCXA pipeline (https://github.com/ebi-gene-expression-group/scxaworkflows) to process in excess of 4 million cells to build a large cross-species collection of single cell expression profiles [22]. 


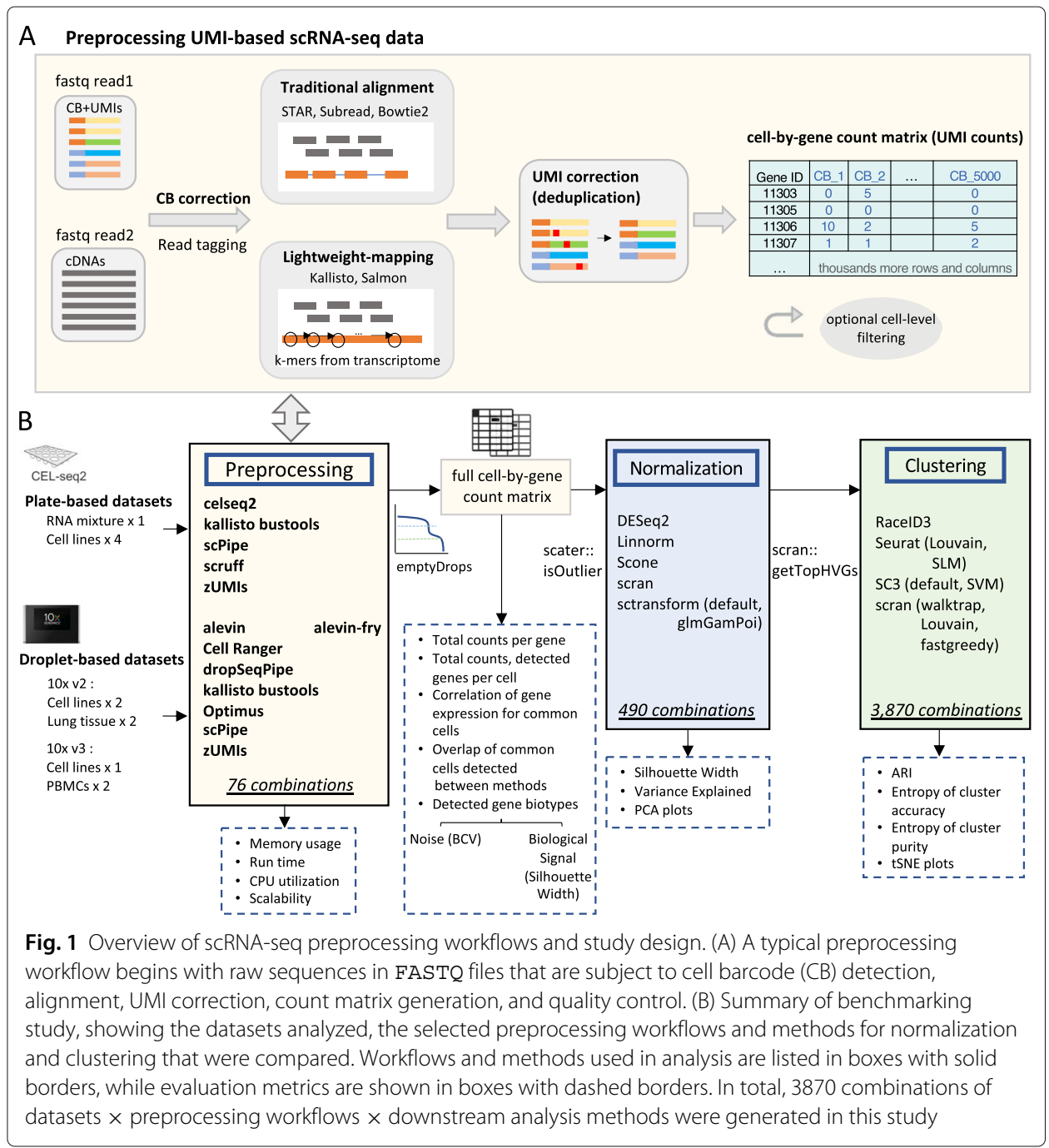

Differences between published workflows arise when researchers balance efficiency with accuracy at each of the abovementioned steps. For example, to obtain CBs, most workflows use an allow list as a reference, whereas salmon alevin generates a putative list of highly abundant CBs that can be further filtered. To deduplicate UMIs, kallisto bustools [23] applied a naive collapsing strategy that they found to be more effective than more complicated approaches. Other pipelines such as Cell Ranger and zUMIs also take base quality and edit distance into consideration during UMI deduplication. Methods that place more importance on this step have also been developed after finding edit distances were inadequate for dealing with UMIs with high similarity [24, 25]. For example, UMItools introduced a network-based graph approach for this step, alevin [24] constructed parsimonious UMI graphs and dropEst [25] developed a Bayesian method to model UMI errors. To assign multi-mapped reads, several workflows, including Cell Ranger, Optimus, dropSeqPipe, and kallisto bustools discard them, while others treat ambiguous reads in different ways, assigning them to potential mapping positions probabilistically or via other strategies. 
The small number of studies that have compared the performance of scRNA-seq preprocessing algorithms stops at the point before normalization after observing high correlations between count matrices obtained from different workflows or other custom combinations of methods for each step [17, 26]. Quantification differences were studied by comparing the performance of various popular alignment methods and annotation schemes [27] to help guide the choice of such tools. One study compared the performance of high-throughput scRNA-seq pipelines before and after normalization followed by clustering and differential expression analysis [28]. They pointed out a confounding factor akin to batch effects after integrating matrices processed by multiple different workflows applied to the same dataset. However, they did not utilize datasets with gold standard ground truth, and lightweight-mapping workflows such as kallisto bustools and salmon alevin were not included in the comparison. Two more recent studies $[29,30]$ compared the performance of the lightweight-mapping pipelines bustools and alevin-fry [31] in pseudoalignment mode. While their findings on the best-performing method in terms of running time and memory usage disagreed, they both concluded that similar downstream results were produced by these two approaches. In contrast, others found that kallisto bustools detects more cells with low gene content which are likely due to mapping artifacts [32], and pseudoalignment strategies can generate a number of false positive genes per cell [33]. Such spurious assignment was illustrated to be more severe when applying pseudoalignment and can be largely eliminated when structural constraints are applied, or when selective alignment is used [31,33] as illustrated in salmon. Preprocessing tools have also been shown to influence the results of RNA velocity analyses [34] which highlights the potential for downstream effects driven by preprocessing algorithm choice. It is worth noting that previous comparative studies mostly focus on preprocessing for droplet-based protocols thus ignoring plate-based platforms, like CEL-Seq [11] and CEL-Seq2 [35] which are frequently used in some settings.

Here, we systematically benchmarked 10 end-to-end preprocessing workflows, including scPipe, zUMIs, kallisto bustools, dropSeqPipe [36], Cell Ranger, Optimus, salmon alevin, alevin-fry, celseq2 [35] and scruff [37]. Among them, celseq2 and scruff are specific to data generated by CEL-Seq and CEL-Seq2 protocols. Cell Ranger was developed for use with the 10x Chromium platform and is the standard workflow for 10x datasets. DropSeqPipe is only available for droplet-based protocols and is an unpublished online workflow with an instructional video on YouTube that teaches users how to run it. Alevin is a tool integrated within salmon that proposes new methods to handle UMIs and ambiguous reads. Alevin-fry is a successor to alevin that provides more options for mapping, cell detection, and quantification. scPipe, zUMIs, salmon alevin, and kallisto bustools can all handle raw data from both plate and droplet-based platforms, and the first three can also deal with Smart-Seq [38] (a full-length protocol that is not UMI-based) data.

We apply these methods to various scRNA-seq datasets with available ground truth containing varying biological complexity levels to benchmark their performance. Specifically, we describe the basic features of the count matrix produced by each preprocessing workflow and explore the impact on downstream analysis by evaluating the performance of combinations of preprocessing workflows together with various normalization and clustering methods using the CellBench platform [39]. 


\section{Results}

Benchmarking scRNA-seq preprocessing workflows

sCRNA-seq preprocessing workflows evaluated

We investigated the performance of 10 end-to-end workflows applied to plate-based (CEL-Seq2) and droplet-based (10x Chromium v2 and v3 chemistry) data. To process CEL-Seq2 data, we applied celseq 2 and scruff (only applicable to plate-based protocols) along with scPipe, zUMIs, and kallisto bustools (applicable to both plate- and dropletbased protocols). For the 10x data we applied dropSeqPipe, Cell Ranger, Optimus, salmon alevin, alevin-fry, scPipe, zUMIs and kallisto bustools. Here salmon alevin was run using selective alignment to full genome decoys [40]. The alevin-fry method was run in pseudoalignment mode to the splici (spliced + intronic) reference [31]. Details of each workflow and the specific strategies applied during preprocessing are listed in Additional file 1: Table S1.

\section{sCRNA-seq datasets used for benchmarking}

The scmixology datasets [5], which were designed for scRNA-seq benchmarking studies, include cells from distinct cell lines and provide ground truth in various forms (e.g., known clusters based on the mixing strategy applied or based on genetic variation between cell lines). These datasets involve experimental designs that use controlled mixtures of RNA (RNA mixture, $1 \times 384$-well plate of CEL-Seq2 data) to create "pseudo-cells," or actual single cells from up to 5 human lung adenocarcinoma cell lines $(4 \times 384$-well plates of CEL-Seq2 data and $2 \times 10 x$ Chromium v2 datasets). A new dataset using the same five cell lines profiled with 10x Chromium v3 chemistry was also generated (data available from GEO under accession number GSE154870). Other datasets included in our analysis were from the Tabula Muris project [41]. Cells from mouse lung tissue profiled by 10x Chromium $(2 \times 10 x$ Chromium v2 datasets $)$ were included to assess performance in a setting with more cellular diversity than the scmixology datasets. The final datasets which also included more cellular diversity were the $5 \mathrm{k}$ and $10 \mathrm{k}$ peripheral blood mononuclear cells (PBMCs) from healthy donor samples profiled using the 10x Chromium v3 chemistry. These data were downloaded from the 10x Genomics website. A summary of the datasets used, including the number of cells, expected number of clusters, and data structure is given in Additional file 2: Table S2.

Cell labels provided by the scmixology datasets were generated with intermediate BAM files created by scPipe based on single-nucleotide polymorphisms (SNPs) information for the cell line datasets and via labels available from the plate annotation for the mixture experiments. Cells from the Tabula Muris samples were manually annotated using canonical marker genes according to the approach described by the Tabula Muris Consortium (2018) [41]. Cells from the PBMC samples were manually annotated to specific cell types based on canonical immune cell markers (see the "Methods" section) using the same annotated cell types identified in another benchmarking study [33]. These annotated cell type labels were used as the ground truth in our study.

\section{Benchmarking workflow}

An overview of our benchmarking study design is presented in Fig. 1 (B). We generated a cell-by-gene count matrix using each of the preprocessing workflows listed in Additional file 1: Table S1. We performed cell-level quality control by firstly applying emptyDrops to distinguish empty droplets and cells (droplet-based protocols) and then using 
scater to identify and remove low-quality cells by setting a data-driven threshold on various quality control metrics (applied to both plate- and droplet-based protocols). Count matrices were normalized by six representative normalization methods that included scran, Linnorm, scone, DESeq 2 and sctransform (in either standard mode or using the method from the glmGamPoi [42] package). We then selected highly variable genes (HVGs) and applied up to eight commonly used clustering methods, including RaceID3, Seurat with the smart local moving (SLM) or Louvain algorithms, SC3 in either default mode or with SVM, and scran with the walktrap, Louvain, or fastgreedy algorithms.

Overall, 3870 different combinations of datasets $\times$ preprocessing workflows $\times$ downstream analysis methods were obtained, with performance evaluated by several metrics at each step (see the "Methods" section and Fig. 1 (B)). CellBench was used to compare these combinations at the pipeline level, which allowed us to assess both the performance of a single method at a specific processing step and the interaction of multiple methods across several steps.

\section{Comparing computational performance of scRNA-seq preprocessing workflows}

As single-cell technology develops, anywhere from hundreds to tens of thousands of cells are routinely profiled in an experiment. An important consideration when choosing between preprocessing workflows is their requirement of time and memory. Assessing how well different tools scale to datasets comprising of very large cell numbers when given more resources is another area of interest.

We compared workflows by specifying one node and eight threads on a HighPerformance Computing (HPC) system. Maximum memory and time requirements were set for each submission. To control for competing workloads on the HPC, we ran each preprocessing method on datasets of different sizes three times per dataset and recorded run time, maximum memory usage, and CPU utilization (see the "Methods" section for details). A summary of the results obtained is shown in Fig. 2. For plate-based protocols, scruff required more memory and was slower when data volume increased (Fig. 2A), which is in contrast to the results of Wang et al. [37]. We speculate that this discrepancy is due to their use of smaller datasets containing fewer than 10 million reads and differences in the hardware and parallelization settings used for evaluation. scPipe, zUMIs and celseq 2 showed similar maximum memory consumption, running times and CPU utilization (Additional file 3: Figure S1A).

For droplet-based protocols, zUMIs was unable to provide results on FASTQ files sampled from the 10xv3_pbmc10k dataset with 600M reads within 2 days and had more variability in run time compared to other workflows on datasets that it did recover results for (Fig. 2B). Workflows based upon pseudoalignment tools required less memory resource and ran faster, which is concordant with previous studies $[18,19]$. Kalliso bustools and alevin-fry were more than 50 times faster when dealing with $600 \mathrm{M}$ reads compared to the second slowest workflow dropSeqPipe. Maximum memory usage was at a similar level for the selected workflows except for zUMIs and dropSeqPipe which required considerably more memory. Among them, dropSeqpipe and scPipe used more memory as dataset size (in terms of the number of reads) increased. Salmon alevin and alevin-fry displayed the highest value of CPU utilization (Additional file 3: Figure S1B), indicating less time is spent waiting. Another study [28] also com- 

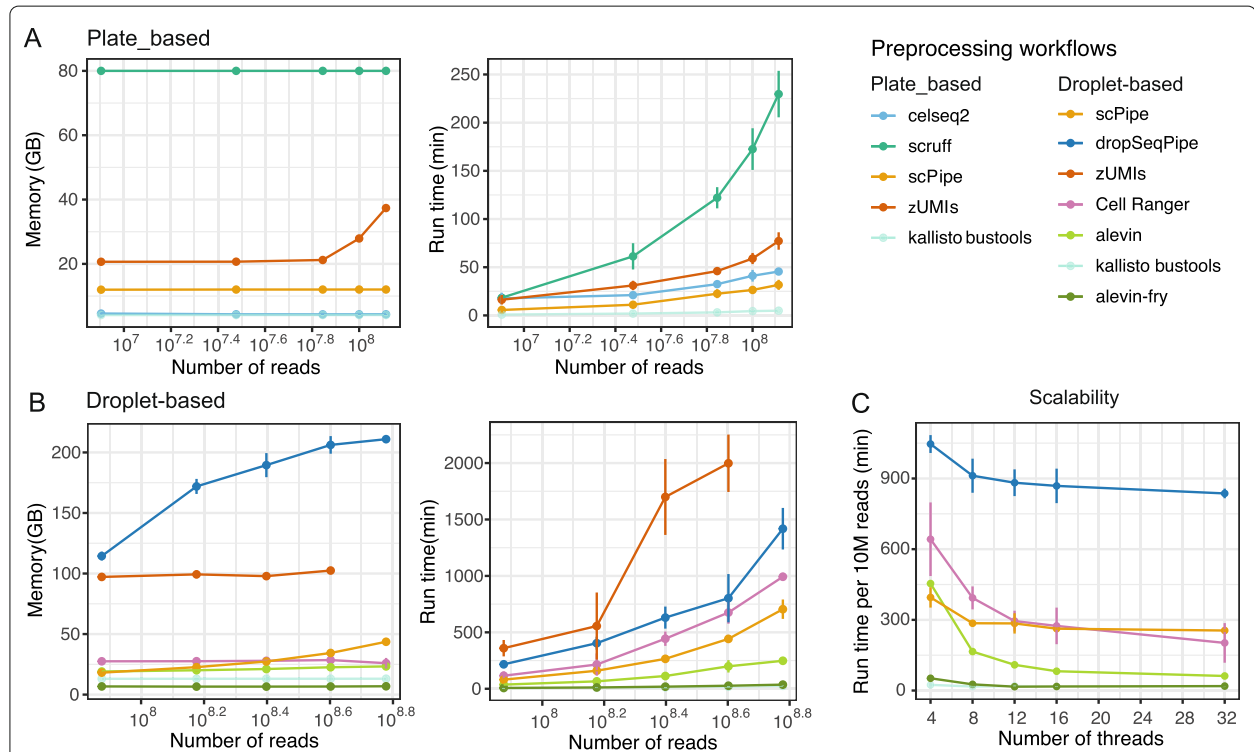

Fig. 2 Comparing the computational performance of different scRNA-seq preprocessing workflows. Maximum memory usage and run time for each preprocessing workflow are shown for $\mathbf{A}$ plate-based protocols and $\mathbf{B}$ droplet-based protocols. Run time versus the number of threads is shown in $\mathbf{C}$, where run time is scaled by 10 million reads

pared the computational performance of preprocessing workflows and demonstrated higher CPU utilization values and shorter running times for Cell Ranger, which is contrary to our results. This difference is likely due to our specification of a fixed number of cores and a limit on maximum memory usage for evaluation, whereas Gao et al. [28] did not.

In terms of scalability, workflows were run on the 10xv3_pbmc5k datasets, and the run time was scaled by $10 \mathrm{M}$ reads. As shown in Fig. 2C, Cell Ranger and dropSeqPipe displayed decreasing trends from 16 to 32 threads, suggesting they have better scalability, while the processing speed of alevin-fry saturated at 16 threads (Additional file3: Figure S1C) and scPipe and kallisto bustools saturated at 8 threads, which is consistent with another study [33].

\section{Comparing gene quantification across scRNA-seq preprocessing workflows}

Besides computational efficiency, the characteristics and accuracy of the biological information recovered by different methods is another key consideration when selecting a preprocessing workflow. Using the cells retained after cell-level quality control (see the "Methods" section) and genes with overall expression above zero (i.e., a count of one or more in at least one cell), we characterized the workflows in terms of the number of genes detected per cell, total counts per cell, correlation of gene expression between common cells, and the concordance of retained cells identified by different workflows.

\section{Gene quantification for CEL-Seq2 workflows}

For the CEL-Seq 2 benchmarking datasets, 5 preprocessing workflows were applied: scruff, celseq2, scPipe, zUMIs, and kallisto bustools. There was little variation in the different metrics assessed between CEL-Seq2 datasets, so representative results for the plate_3cellline dataset are shown in Fig. 3. 


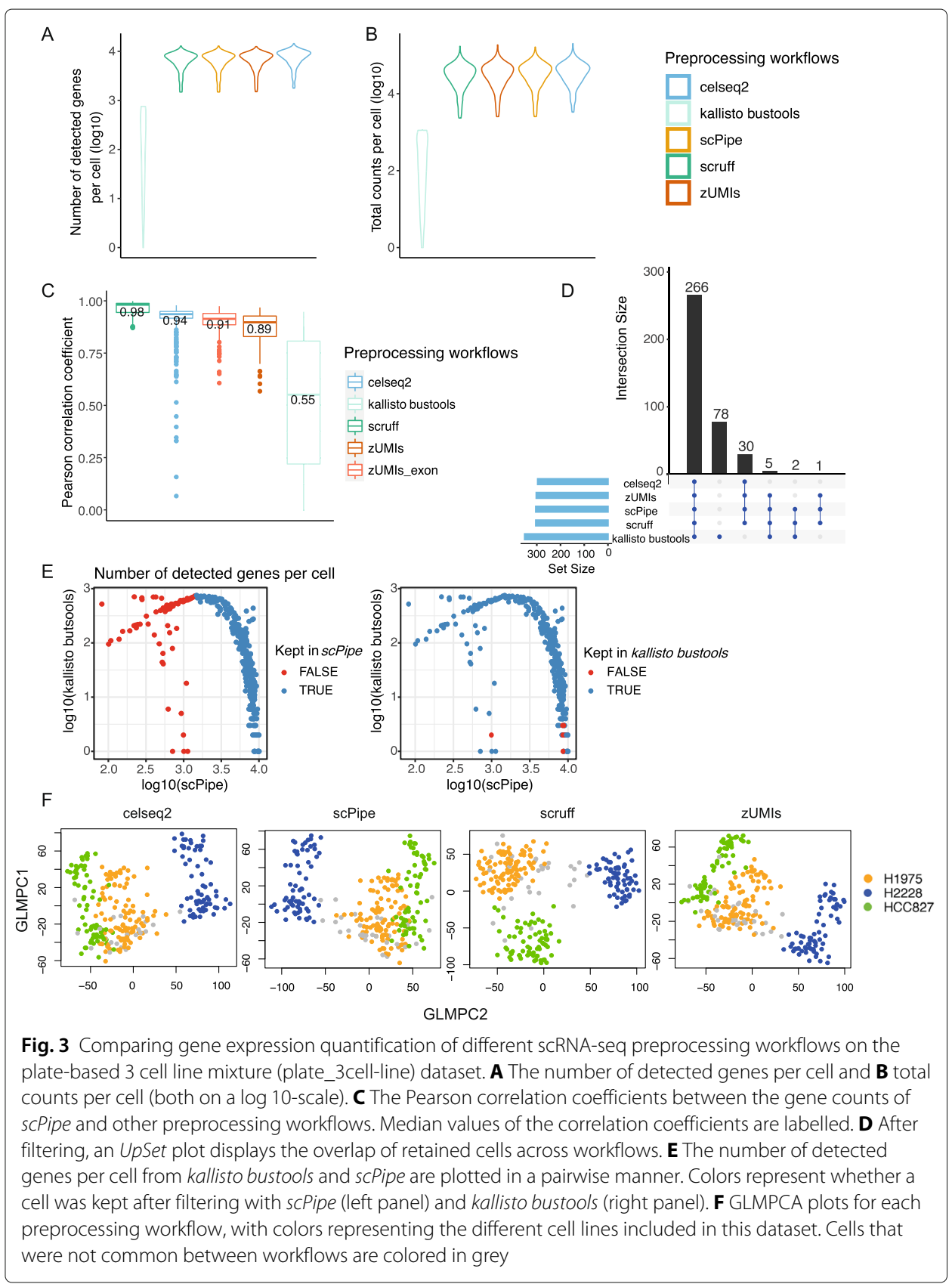

In summary, the number of detected genes and total counts per cell showed high similarity across preprocessing workflows except for kallisto bustools (Fig. 3A, B), which recovered fewer genes and lower counts. Upon further investigation, this was found to be due to its strategy of only retaining unique CB-UMI pairs (further details on this issue can be found at https://github.com/BUStools/bustools/issues/44). For short UMIs (6 bp in the case of the CEL-Seq2 datasets), this limits the maximum detected features per cell to $4^{6}=4,096$ ( 3.61 on the $\log _{10^{-}}$scale). In practice, the UMI counts are much lower, as whenever the same UMI is observed across more than one gene in a given cell, it is removed from the analysis altogether. This deficiency led us to exclude kallisto bustools from the majority of comparison plots for the CEL-Seq2 preprocessing results. 
Slightly more detected genes per cell were observed with celseq2 (Fig. 3A and Additional file 3: S2A), suggesting higher sensitivity of the aligner it uses (Bowtie2) on the datasets tested. scruff and scPipe were in complete agreement concerning the number of detected genes (Additional file 3: Figure S2A), which is as expected, as they share a very similar strategy across all preprocessing steps except for the quantification method used to count aligned reads. Higher total counts per cell were observed with scPipe compared with those of scruff (Additional file 3: Figure S2B). We speculate that the strategy applied in scPipe of UMI collapsing and read quantification may give rise to fewer collapsed UMIs and more assigned reads, and consequently, higher total counts per cell.

We next investigated the concordance of gene expression across workflows and compared the correlation of gene expression between scPipe and other methods using common cells and genes (Fig. 3C). Overall, we found relatively high average Pearson correlations (nearly all above 0.9), with correlations between scPipe and scruff the highest, whereas, with celseq2 and zUMIs, the correlations were slightly lower (Fig. 3C). Celseq2's use of a different aligner (Bowtie2) might account for the lower correlations, while for zUMIs the inclusion of intron reads in the gene counts is partially responsible, with the correlation increasing when run in exon-only mode. In terms of the overlap in cells detected by different methods, the majority (266) were common across all methods, with a further 38 found by at least 3 out of the 5 workflows tested (Fig. 3D). kallisto bustools detected 78 unique cells, which were deemed to be of low quality by other methods such as scPipe and filtered out due to low numbers of genes detected per cell (Fig. 3E).

Next, we applied GLMPCA [43], an alternative dimension reduction method for visualizing the raw counts from scRNA-seq data (Fig. 3F and Additional file 3: Figure S2C). Clear separation between cells from the different cell lines was observed for all preprocessing workflows, except for kallisto bustools (Additional file 3: Figure S2C). Samples from cell lines H1975 and HCC827 were observed to be more similar according to their bulk expression profiles in a previous study [44], which is broadly consistent with what we observe here at the single-cell level.

\section{Gene quantification for 10x workflows}

The same metrics were applied to the raw count matrix to compare the performance of 8 preprocessing workflows applicable to droplet-based 10x datasets (scPipe, zUMIs, kallisto bustools, salmon alevin, alevin-fry, Cell Ranger, dropSeqPipe, and Optimus) after applying emptyDrops or Cell Ranger v2 filtering (see the "Methods" section).

In terms of the number of detected genes and total counts per cell, zUMIs followed by scPipe systematically recovered more genes and higher counts across datasets (Fig. 4A, B and Additional filer̃efMOESM3: Figure S3A-D), while results of other workflows are relatively concordant, except for kallisto bustools which detected more features on the PBMC datasets. To further explore these discrepancies, we compared the metrics obtained by Cell Ranger versus other workflows in a pairwise manner, with representative results on the 10xv2_3cell-line and 10xv3_pbmc5k datasets shown in Fig. 4C, D and Additional file 3: Figure S4A-B respectively. We summarized the differences in terms of whether there is a systematic overestimation or underestimation and the slope and variation of the linear relationships. Regarding the number of features detected, similar to the results of overall distribution of these metrics (Fig. 4A), zUMIs, followed by scPipe, salmon alevin on 10xv2_3cell-line, and kallisto bustools on 10xv3_pbmc5k recovered more features 

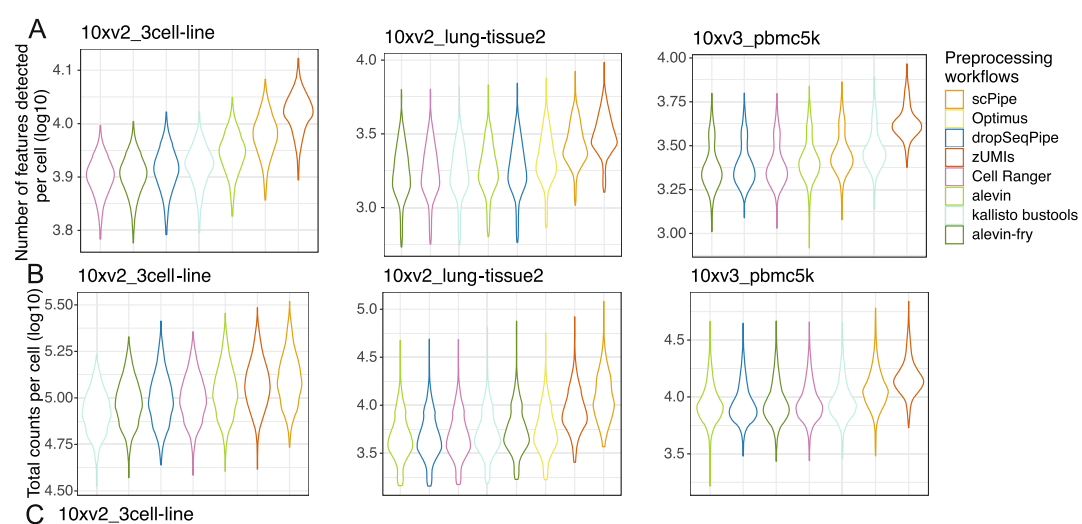

C 10xv2_3cell-line

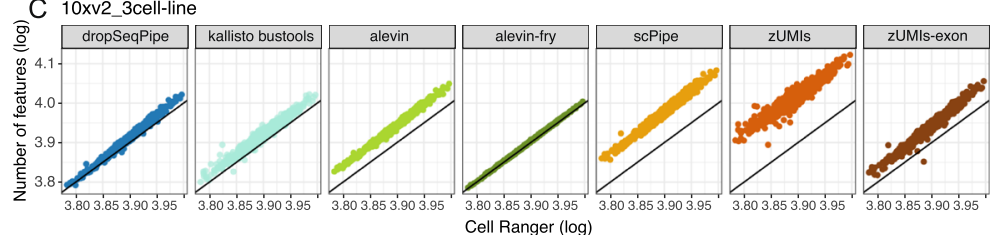

D 10xv2_3cell-line Cell Ranger (log)
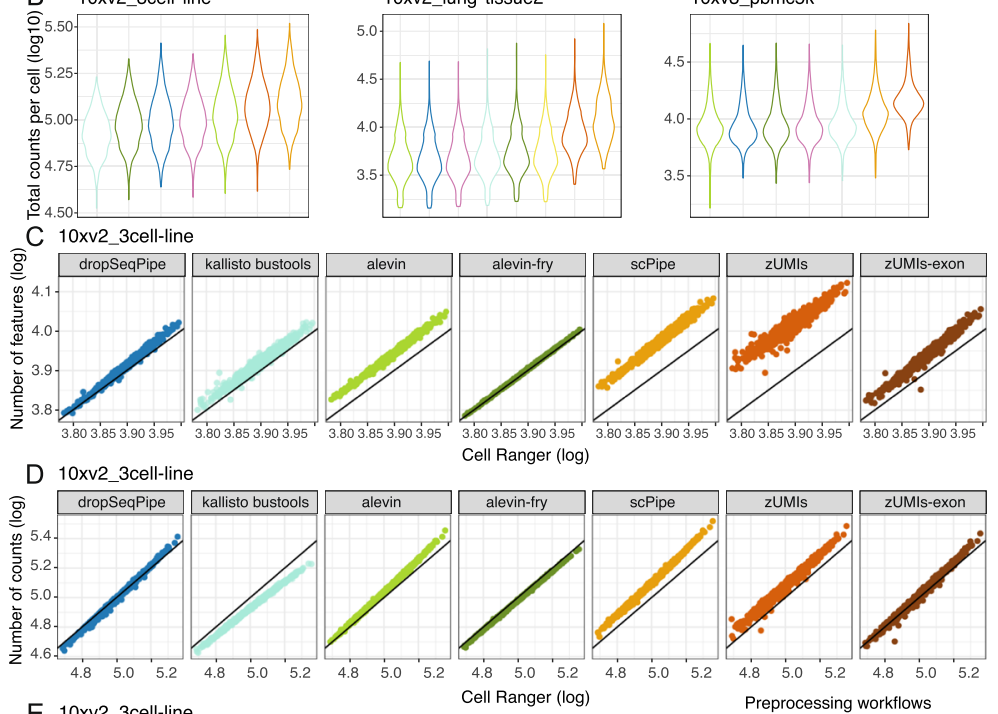

Preprocessing workflows

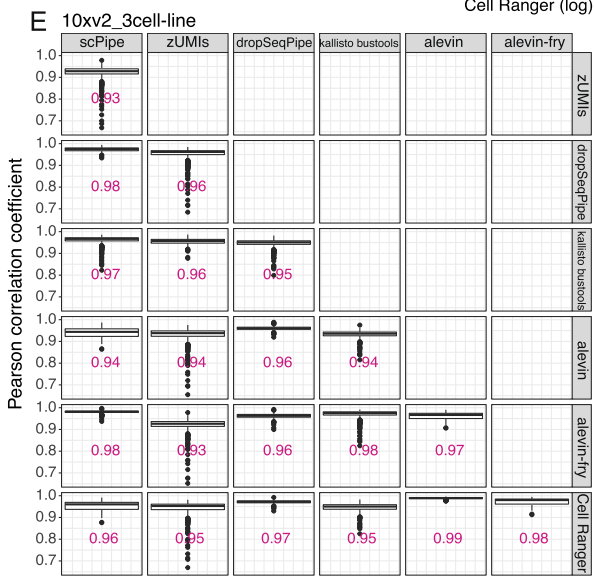

- dropSeqPipe

- dropSeqPipe

$\begin{array}{lll}\text { - zUMls } & \text { alevin-fry } \\ \text { - Cell Ranger } & \text { zumis_exon }\end{array}$

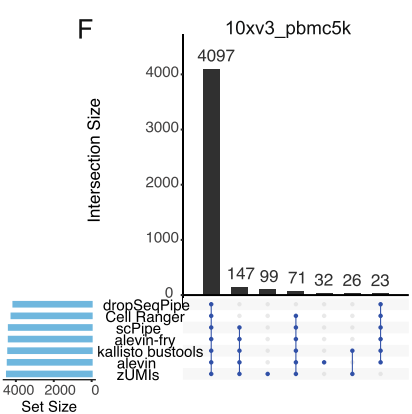

Fig. 4 Comparing gene expression quantification of different scRNA-seq preprocessing workflows on droplet-based datasets. A The number of detected genes per cell and $\mathbf{B}$ total counts per cell (both on a $\log 10$-scale) on the 10xv2_3cell-line, 10xv2_lung-tissue2, and 10xv3_pbmc5k datasets. C The number of detected genes per cell and $\mathbf{D}$ total counts per cell for common cells for different preprocessing workflows against Cell Ranger on the 10xv2_3cell-line dataset. The identity line $(y=x)$ is plotted in black in each panel. E The Pearson correlation coefficients between the gene counts of different pairs of preprocessing workflows for the 10xv2_3cell-line dataset. Median values of the correlation coefficients are labelled. F An UpSet plot displays the overlap of retained cells across workflows on the 10xv3_pbmc5k dataset

compared to Cell Ranger. Moreover, zUMIs and kallisto bustools displayed more variation from the fitted linear relationship. Including intron reads partially explained the extra detected features, as well as its variation (right-most panel Fig. 4C) for $z U M I s$, while for other workflows, applying a full reference annotation and different aligners should account for the extra genes detected compared to Cell Ranger, which uses a curated annotation. In terms of total counts per cell (Fig. 4D and Additional file 3: Figure S4B), zUMIs, 
followed by scPipe showed overestimation relative to Cell Ranger as well. Besides this, they generally recovered relatively more counts in cells with a higher abundance as quantified by Cell Ranger (slope of the fitted linear relationship above 1). This was also found with salmon alevin, dropSeqPipe, and zUMIs_exon on the 10xv2_3cell-line dataset. In contrast, kallisto bustools recovered higher counts in cells with lower content as quantified by Cell Ranger (slope below 1), which might be related to its naive UMI collapsing strategy. Interestingly, although alevin-fry uses a pseudoalignment approach, it provides the most concordant results with the least variation when compared against the Cell Ranger results.

Calculation of the Pearson correlation of expression for common cells across workflows showed high average pairwise concordance for all workflows, especially between salmon alevin, alevin-fry, dropSeqPipe, and Cell Ranger (Fig. 4E and Additional file 3: Figure S4C), with median correlation consistently above 0.95. alevin-fry also displayed a high correlation with kallisto bustools, which might be on account of them both applying pseudoalignment strategies. However, zUMIs showed relatively lower correlations with other workflows. Calculating correlations with intron counts excluded noticeably increased the correlation values (Additional file 3: Figure S4D), which suggests that the addition of intron counts is potentially adding noise to the results.

Next, UpSet plots were generated to assess the concordance of retained CBs after filtering across workflows (Fig. 4F and Additional file 3: Figure S4E). We found that most of the retained cells were common across workflows, and cells that were unique to a subset of the workflows made up less than $5 \%$ of the total number of cells.

\section{Comparing gene biotype detection across scRNA-seq preprocessing workflows}

Genes of different biotypes can have systematically distinct length distributions and sequence similarity [45] and current RNA-seq tools have been shown to quantify genes possessing these characteristics differently [46, 47]. To explore differences in the detection and quantification of gene biotypes across scRNA-seq workflows, we investigated the signal and noise characteristics stratified by biotype.

\section{Gene biotype detection for CEL-Seq2 workflows}

For the representative plate_3cell-line dataset, density plots of the total counts per gene (Fig. 5A) were similar for all methods, except for kallisto bustools. To compare the biological noise for each workflow, the biological coefficient of variation (BCV) was calculated using the known cell labels from each dataset as the ground truth (see the "Methods" section). $\mathrm{BCV}$ measures the proportion of gene expression attributable to biological variability [48] and typically starts from higher values at low abundance and decreases monotonically as gene abundance increases. We observed such trends for most listed preprocessing workflows with selected biotypes using all cells and all features (Fig. 5B). The $\mathrm{BCV}$ trend for scPipe was systematically higher (especially for low intensity features) than the trends for other methods which are more consistent (results for kallisto bustools were excluded from this plot due to the limited numbers of features available to estimate $\mathrm{BCV}$ reliably). In terms of detected biotypes, the highest sensitivity and most lncRNAs were obtained with celseq2, which also detected features in the misc_RNA class (Fig. 5C), while zUMIs detected fewer pseudogenes compared to other methods. No major differences 


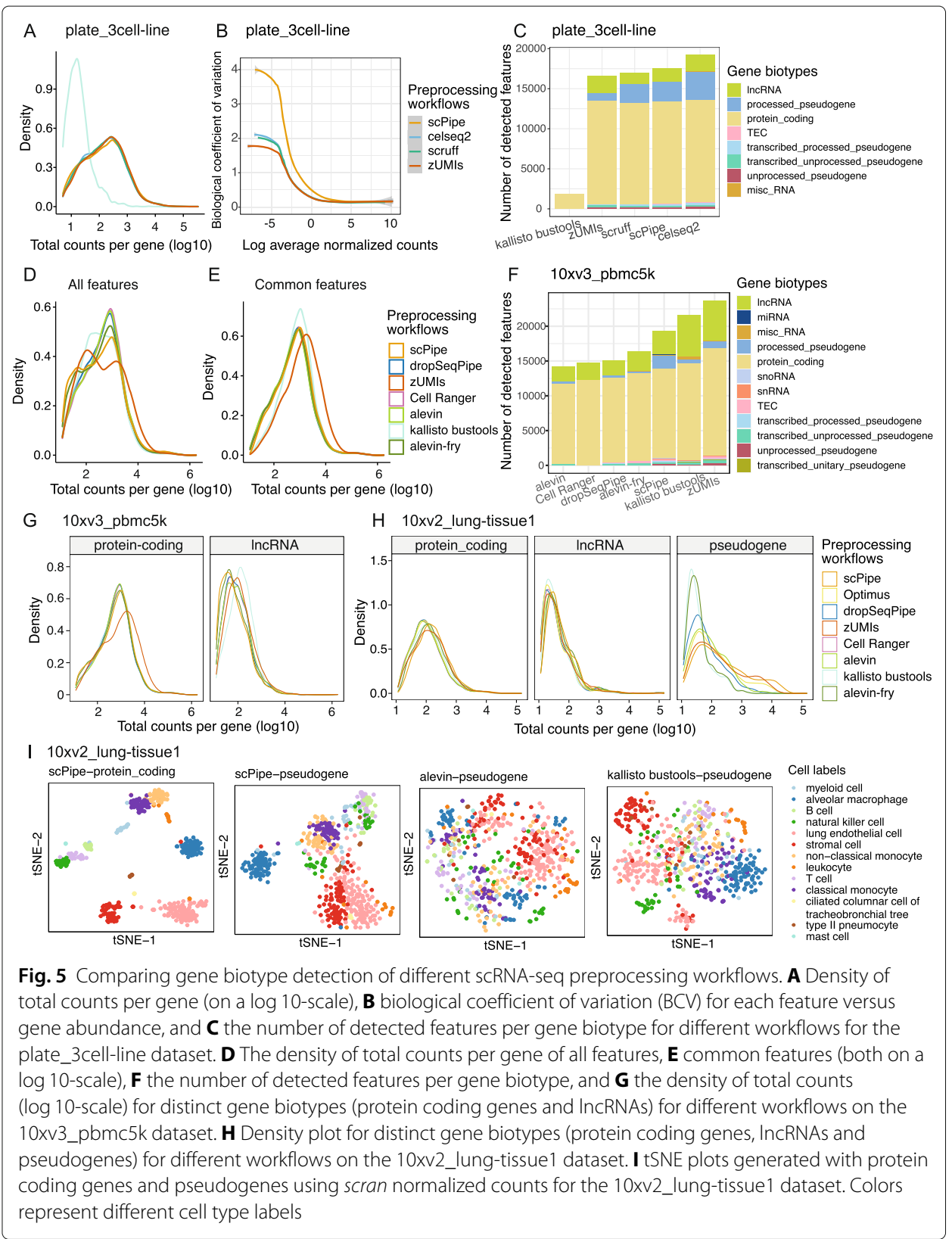

were observed in the count distributions of the various gene biotypes between different preprocessing methods (Additional file 3: Figure S5A).

\section{Gene biotype detection for 10x workflows}

Results for the droplet-based workflows (the 10xv3_pbmc5k dataset was chosen as a representative example in Fig. 5D and Additional file 3: Figure S5B, with other datasets featured in Additional file 3: Figure S5C-G) show bimodal gene count distributions for most datasets, with the exception of the lung tissue dataset (Additional file 3: Figure S5C) which has a unimodal count distribution. Cell Ranger, together with salmon alevin consistently detected fewer genes at the lower peak and more genes at the higher peak, whereas other methods provided relatively fewer features in the higher peak (Fig. 5D and 
Additional file 3: Figure S5D-G left panel). zUMIs and kallisto bustools tended to recover more lowly expressed genes across datasets, with kallisto bustools having a flatter density (on 10xv3_pbmc5k, 10xv3_pbmc10k, 10xv2_5cell-line) spread between the first and second peak observed using other methods.

If we restrict our analysis to features that are common across workflows, the lower peak is less prominent (Fig. 5E and Additional file 3: Figure S5D-G right panel), suggesting that the features quantified with low abundance tend to be discordant between workflows, which is in agreement with previous results [46]. After filtering the data in this way, the zUMIs peak is shifted from the left to the right and the density peak for kallisto bustools is much narrower.

We next studied the number of detected genes and proportion of counts assigned and found that they varied across different biotypes between workflows, with some workflows more likely to detect or assign counts to a particular class of genomic features than others (Fig. 5F and Additional file 3: Figure S6A). Most of the counts are assigned to protein coding genes across workflows (Additional file 3: Figure S6A). Cell Ranger's use of a curated reference annotation restricts analysis to lncRNAs and protein coding genes, while scPipe, followed by zUMIs and Optimus assigned fewer counts to protein coding genes and a greater proportion of counts to pseudogenes. Kallisto bustools detected more lncRNAs (Fig. 5F) and generally assigned more reads to this class of features (Additional file 3: Figure S6A). Although short non-coding RNAs, including miRNAs, small nuclear RNAs (snRNA), and small nucleolar RNAs (snoRNA) were detected at very low levels $(<0.2 \%$, Additional file 3: Figure S6A), scPipe, zUMIs, and Optimus assigned a relatively higher percentage of UMI counts to these biotypes compared to other methods. The different biotypes influence the count distribution, with lncRNAs and pseudogenes contributing more to the lower abundance peak and protein coding genes to the higher abundance peak (Fig. 5G, H). Protein coding genes have similar densities, with a peak in the high abundance range across most methods except for $z U M I s$, where the peak is shifted to the right (Fig. 5G left panel and Additional file 3: Figure S6B-C left panel). This shift is also present for lncRNAs detected by zUMIs on the PBMC datasets (Fig. 5G right panel and Additional file 3: Figure S6B middle panel) and is presumably caused by the systematic inclusion of intronic and exonic reads when it assigns them to features. kallisto bustools shows a peak with higher abundance of lncRNAs as well on the PBMC and cell line datasets (Fig. 5G right panel and Additional file 3: Figure S6B-C middle panel), which might be the cause of its distinct distribution of total counts per gene observed when using all features (Fig. 5E and Additional file 3: Figure S5D-G, left panels).

The pseudogene total count per feature distributions were the most variable between workflows, with kallisto bustools, and alevin-fry having the sharpest left-skewed peak, suggesting more lowly expressed pseudogenes with fewer than 10 counts were quantified by these methods (Fig. 5H and Additional file 3: Figure S6B-C right panels). For other workflows, the peak is shifted to the right to varying degrees, indicating the existence of workflow-specific quantification biases for pseudogenes.

To further evaluate the quantification performance of selected workflows on pseudogenes, we examined long-read transcriptome sequencing data on the same cell line mixture samples [49] to obtain an independent measure of pseudogene abundance in the 10xv2_5cell-line and 10xv3_5cell-line datasets. Longer reads should map less ambigu- 
ously to pseudogenes compared to short-read data [50,51], so the proportions obtained should be closer to the truth. Comparing the proportions of counts mapped to pseudogenes from the different preprocessing methods to those obtained in the long-read data, which themselves varied between the $10 \mathrm{x} v 2$ and v3 chemistry, we consistently observed fewer counts being assigned by dropSeqPipe, salmon alevin (selective alignment), and the pseudoalignment tools kallisto bustools and alevin-fry, which suggests that these workflows systematically underestimate pseudogene abundance (Additional file 3: Figure S6D-E). zUMIs recover pseudogene count proportions that are similar to the long-read estimates, while scPipe systematically assigns more reads to pseudogenes and is probably overestimating signal in this class of features.

\section{Comparing noise and biological signal across gene biotypes}

Given the differences in detecting specific biotypes across the 10x preprocessing workflows compared, we next investigate the biological information captured by the 3 most abundant classes, which are protein coding genes, lncRNAs and pseudogenes (Additional file 3: Figure S6F). BCV and silhouette widths were calculated using the known cell type labels from each dataset as the ground truth (see the "Methods" section) to compare the biological noise and signal across biotypes for each workflow, respectively.

We observed expected $\mathrm{BCV}$ trends (i.e., BCV decreases as abundance increases) for all listed preprocessing workflows for the selected biotypes using common cells and all features (Additional file 3: Figure S7A-C left panel). BCV trends were similar for protein coding genes and lncRNAs for most of the datasets, while higher variance of lowly expressed genes were observed by kallisto bustools, alevin-fry, and scPipe on the lung tissue data; zUMIs on cell line data; and scPipe, alevin-fry, and zUMIs on the PBMC data. The trends were fairly distinct for the pseudogenes, especially for kallisto bustools and alevin-fry on the lung tissue data (Additional file 3: Figure S7A right-most side of left panel) and salmon alevin on the cell line data (Additional file 3: Figure S7B right-most side of left panel) where the $\mathrm{BCV}$ was systematically higher across the full range of abundance levels. The pseudogenes BCV trends for kallisto bustools and alevin-fry was markedly higher for the low abundance features on the PBMC datasets only (Additional file 3: Figure S7C right-most side of left panel). Restricting the analysis to common features and cells detected across all workflows saw similar trends (Additional file 3: Figure S7A-C right panel), although $\mathrm{BCV}$ values decreased overall compared to the results obtained from all features (Additional file 3: Figure S7A-C left panel), suggesting that quantification of discordant features is a major source of variation between workflows.

Next, we investigated the biological signal recovered by features from specific biotypes. Silhouette widths calculated on GLMPCs, which measures how similar a cell is to its "known" (pre-labeled) cell type compared to other cells were used and compared (see the "Methods" section). For the 10x cell line, lung tissue and PBMC datasets, protein coding genes showed similar and higher silhouette widths, indicating biological signal was universally retained by all workflows (Additional file 3: Figure S8A).

The separation between different cell types can be visualized using t-SNE plots created using protein coding genes (Fig. 5I left-most panel and Additional file 3: Figure S8B-C left-most panel). Most lncRNAs and pseudogenes had silhouette widths above 0 for the cell line datasets (Additional file 3: Figure S8A left column). However, on datasets with more cell type complexity, silhouette widths consistently above 0 were only observed 
for lncRNAs with $z U M I s$ and for pseudogenes with scPipe, Optimus, and $z U M I s$ on the lung tissue data (Additional file 3: Figure S8A middle column); lncRNAs with alein-fry, dropSeqPipe, kallisto bustools, and pseudogenes, with scPipe and zUMIs on the PBMC data (Additional file 3: Figure S8A right panel), suggesting that the separation between the known cell groups was less well defined by these feature types. Kallisto and salmon were recommended for detecting lncRNAs in a previous bulk RNA-seq benchmarking study [52] and although on the PBMC data we observe that kallisto bustools, alevin, alevin-fry, and dropSeqPipe recover more biological signal for lncRNAs than other methods, which is concordant with these findings, this was not seen in other datasets (Additional file 3: Figure S8A top row).

Additionally, looking at t-SNE plots based on pseudogenes for salmon alevin, kallisto bustools, and alevin-fry (Fig. 5I and Additional file 3: Figure S8B-D) shows a less clear separation between cell types compared to those provided by other workflows, especially on the cell line (Additional file 3: Figure S8C) and lung tissue datasets (Additional file 3: Figure S8D). This suggests that the quantification of pseudogenes by pseudoalignment or selective alignment using 3' short-read sequencing data recovers less biological information. These results suggest that focusing analysis efforts on the signal from protein coding genes in datasets that profile complex tissues with greater cell type diversity may be an optimal strategy for all preprocessing methods.

\section{Comparing the effects of preprocessing workflows on downstream analysis}

We next examined the degree to which the choice of processing workflow influences downstream analysis. Specifically, we look into how preprocessing impacts normalization, highly variable gene (HVG) selection, and clustering.

\section{Comparing the performance of different combinations of preprocessing workflows and normalization methods}

Normalization methods and evaluation metrics Normalization has been shown to be an influential step in previous benchmarking studies [27, 53]. We applied six popular and well-proven normalization methods [5, 54], including DESeq2 [55], scone [54], scran [56], Linnorm [57], and sctransform [58] (both the default method and updated strategy which uses glmGamPoi [59]), to explore how well different approaches remove any of the inherent biases introduced by preprocessing.

We evaluated the performance of each combination of dataset $\times$ preprocessing method $\times$ normalization algorithm using the silhouette widths of known cell groups, along with the amount of unwanted variation explained by library size and wanted variation explained by known cell groups on principal components (PCs). To summarize the results across the many different combinations of methods, linear models were fitted with silhouette widths as the response variable and the different methods as covariates. Higher silhouette widths and reduced unwanted variation are both indicators of better performance (see the "Methods" section). PCA plots were also generated for each combination of preprocessing workflow and normalization to assist in visualizing the expected data structure.

Normalization performance assessment on CEL-Seq2 datasets Considering silhouette widths on the CEL-Seq2 RNA mixture datasets, no preprocessing workflow sys- 
tematically outperformed others when combined with different normalization methods (Fig. 6A left column). Example PCA plots are shown in Additional file 3: Figure S9A, with most of the combinations presented showing the expected trajectory paths, and combinations evaluated with higher silhouette widths displaying better separation between distinct RNA mixtures, e.g., for scone, scruff followed by scPipe, zUMIs, and celseq2. In terms of variation explained by library sizes, relatively less variation was explained by scPipe combined with any normalization methods compared to other preprocessing methods, which is preferred (Additional file 3: Figure S9B).

For the cell line datasets, $z$ UMIs had slightly higher silhouette widths relative to other preprocessing methods while celseq 2 had consistently lower silhouette widths on average across different normalization methods (Fig. 6A right column and Additional file 3: Figure S9C). Regarding unwanted variation, performance was fairly similar across the different combinations (Additional file 3: Figure S9D).

Normalization performance assessment on 10x datasets Similar silhouette widths after normalization were observed across workflows on the droplet-based datasets

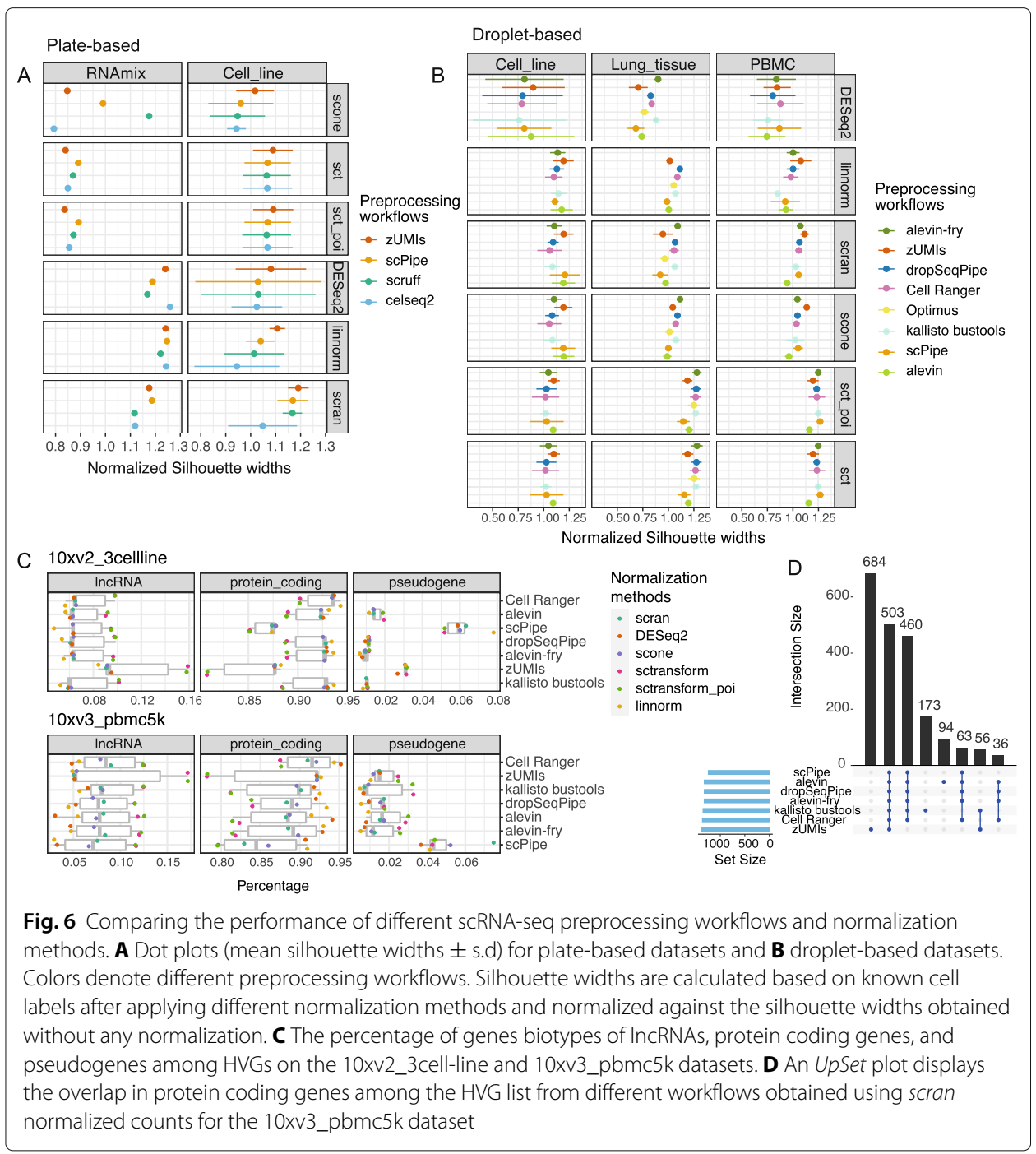


(Fig. 6B). On the cell line datasets, silhouette widths were higher for combinations of scPipe, zUMIs, and salmon alevin with scran or scone normalization. Overall, zUMIs followed by salmon alevin and scPipe provided slightly higher silhouette widths across all normalization methods, while dropSeqPipe, kallisto bustools, and Cell Ranger return relatively lower silhouette widths on the cell line data (Additional file 3: Figure S10A left panel). Inspection of PCA plots (Additional file 3: Figure S10B) from scran-normalized data showed that combinations with higher silhouette widths (scPipe and zUMIs) had better separation of H1975 and HCC827 cells compared to other workflows, although all methods showed good separation overall. In terms of unwanted variation, workflows performed fairly similarly, although zUMIs retained more library size variation post normalization than other methods (Additional file 3: Figure S10C).

On the lung tissue datasets, we observed that the best performing preprocessing methods on the cell line datasets (zUMIs, scPipe, and salmon alevin) tended to have slightly lower silhouette widths, while the other workflows performed fairly similarly, with slightly higher silhouette widths irrespective of the normalization method chosen (Fig. 6B middle column and Additional file 3: Figure S10A middle panel). In terms of unwanted variation, scPipe retained more library size variation than other methods and workflows that applied pseudoalignment (alevin-fry and kallisto bustools) in contrast retained less unwanted variation (Additional file 3: Figure S10D).

On the PBMC datasets, zUMIs performed better in combination with scran, scone, and Linnorm, while scPipe obtained higher silhouette widths when used together with sctransform normalization (Fig. 6B right column and Additional file 3: Figure S10A right panel). With respect to unwanted variation, zUMIs performed better as well with less library size variability, while Cell Ranger and alevin-fry performed relatively worse (Additional file 3: Figure S10E).

Although no preprocessing method consistently ranked the best across the datasets analyzed based on the silhouette widths (Fig. 6B), we found that the ranks of the preprocessing workflows across different normalization methods on datasets of a specific experimental design were relatively stable on average, suggesting that individual preprocessing workflows might incorporate quantification biases that cannot be eliminated by normalization.

Summary of normalization results The performance evaluated by silhouette widths is summarized in Additional file 3: Figure S11. Results from all combinations of different preprocessing workflows and normalization methods show increased silhouette width compared to the results obtained without any normalization, suggesting that all normalization methods are highly effective on these data. In terms of preprocessing workflows, highly similar performance is displayed across methods, with normalization methods performing slightly better with $z$ UMIs on droplet-based datasets, following very closely behind by alevin-fry and dropSeqPipe. On plate-based datasets the results are highly consistent between workflows, with less variation shown with $z U M I s$, followed closely behind by scruff, scPipe and celseq 2 .

\section{Comparisons of HVGs selected on 10x datasets}

After normalization, HVGs were selected for each combination of dataset $\times$ preprocessing method $\times$ normalization algorithm using scran (see the "Methods" section). Although 
the proportion of HVGs of different biotypes varies widely across datasets, protein coding genes account for the largest proportion of HVGs (Fig. 6C and Additional file 3: Figure S12A). Proportions also vary between preprocessing workflows, for instance Cell Ranger includes more protein coding genes than other workflows as would be expected since it excludes other biotypes, while $z U M I s$ includes more lncRNAs and scPipe more pseudogenes. zUMIs also includes more pseudogenes among the HVGs on the cell line data compared to other methods and has similar proportions as Optimus on the lung tissue data.

From the perspective of how the choice of normalization method influences the HVG composition, we observe that more IncRNAs and fewer protein coding genes were included in the HVG lists obtained after applying sctransform irrespective of the preprocessing method, while Linnorm and DESeq2 retained the most protein coding genes and fewest lnRNAs, which is especially pronounced for the PBMC datasets (Fig. 6C and Additional file 3: Figure S12A).

While most of the protein coding HVGs detected were common between workflows, distinct sets of genes of varying sizes were retained by individual preprocessing workflows in different datasets. For instance, on the 10xv3_pbmc5k data, zUMIs, kallisto bustools, and salmon alevin normalized by scran found 684, 173, and 94 unique genes respectively (Fig. 6D), while for sctransform glmGamPoi normalization, 555, 111, and 92 unique protein coding genes were recovered by these workflows (Additional file 3: Figure S12B). zUMIs also returned the largest list of unique lncRNAs after both sctransform glmGamPoi (Additional file 3: Figure S12B) and scran (Additional file 3: Figure S12C) normalization, followed by kallisto bustools. For pseudogenes, there were relatively few common highly variable features ( 8 or fewer, Additional file 3: Figure S12B-C), while scPipe selected the largest number of distinct pseudogenes (33 and 85 respectively) in its HVG list that were not retained by any of the other preprocessing workflows.

\section{Comparing the performance of different combinations of preprocessing workflows and clustering methods}

Clustering methods and evaluation metrics There has been much research focused on the performance of clustering methods in terms of sensitivity of parameters, accuracy, robustness, etc. [53, 60, 61]. Here, we aim to investigate the impact of preprocessing on clustering instead of ranking clustering methods based on their performance. We selected representative clustering methods implemented in $\mathrm{R}$ and evaluated their performance when they reach the expected number of clusters based on the labels available. RaceID [62], SC3 [63], scran and Seurat [64] were methods included. Both classic unsupervised methods and combined Support Vector Machine (SVM) methods in SC3 were used. Graph-based clustering methods have been shown to perform fairly well previously, so scran with algorithms of fast-greedy, louvain, and walktrap and Seurat with louvain and SLM [65] were all included in the evaluation. The entropy of cluster accuracy (ECA), the entropy of purity (ECP) (see the "Methods" section), and adjusted Rand index (ARI) [66] were used for assessment of intra-cluster similarity, purity, and similarity of clustering partition with known clusters, respectively. ANOVA was then applied to assess the relative variation explained by the main analysis steps (preprocessing, normalization and clustering, see the "Methods" section). We also fitted a linear model to evaluate the extent to which specific methods or workflows at each analysis 
step, including preprocessing, normalization, and clustering, influenced the clustering result. ARI, ECA, or ECP were used as dependent variables in this analysis. Considering the intrinsic difference between experimental designs, ANOVA and linear models were fitted to the various combinations of methods separately for datasets with different designs.

Clustering performance assessment on CEL-Seq2 datasets The clustering analysis for the RNA mixture datasets is more challenging compared to the other plate-based datasets due to the inbuilt trajectory paths which produce clusters that are very close to one another.

In terms of ARI, combined with selected normalization and clustering methods, scruff produced more combinations with better performance, while scPipe provided relatively fewer and its average performance was relatively worse on the RNA mixture data (Fig. 7A and Additional file 3: Figure S13A). Combinations applying scran clustering algorithms delivered better results while those applying SC3 have systematically lower ARI values. In terms of the top 3 combinations of entropy obtained for each preprocessing method, scruff tended to deliver better results compared to other workflows (Additional file 3: Figure S13B), and interestingly, no clustering method was observed to be consistently the best, while Linnorm normalization was the most common method among the top 3 combinations across workflows.

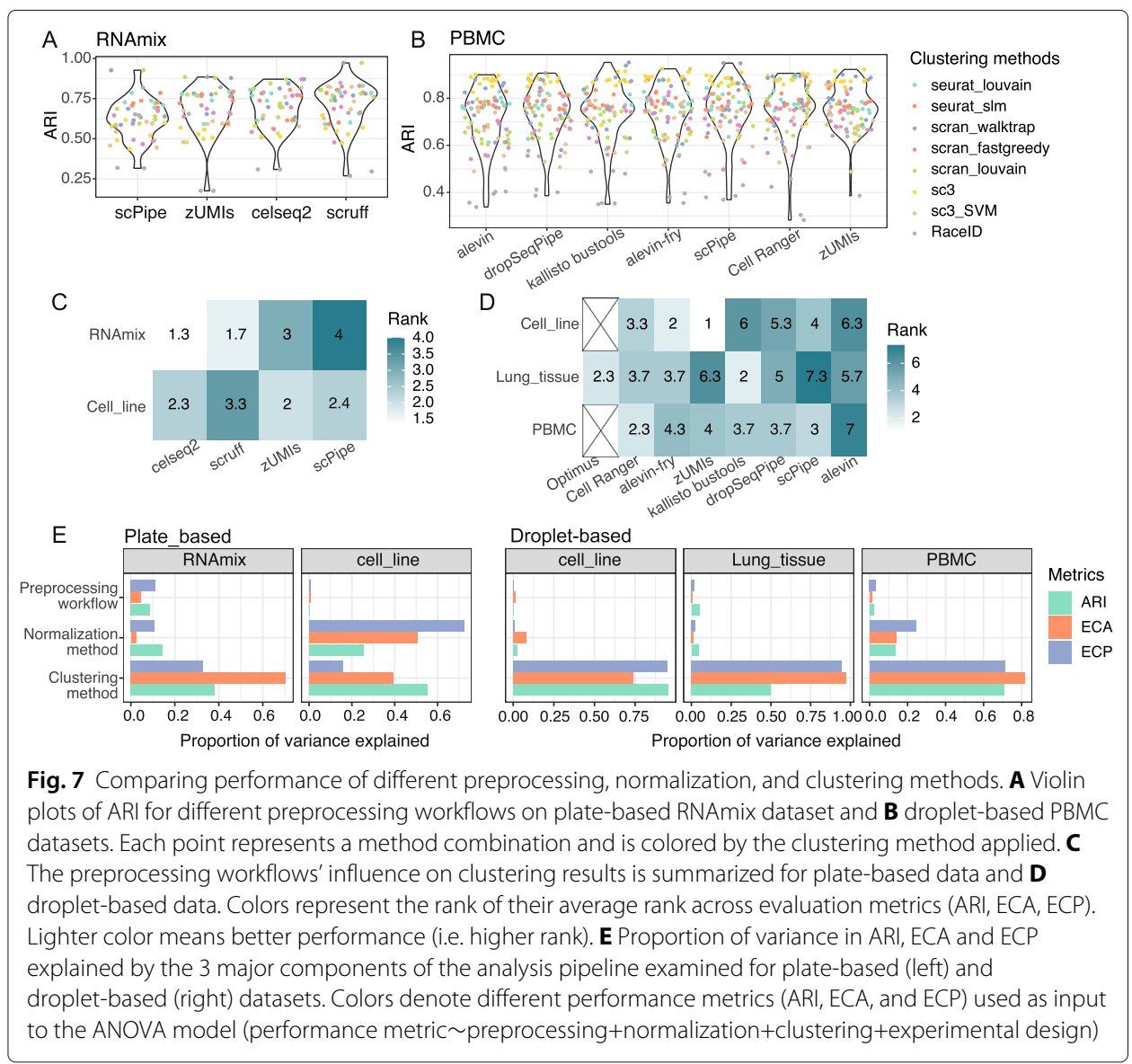


Overall, scruff and celseq2 were ranked first and second across normalization and clustering method combinations according to the coefficients from the linear modeling, indicating better performance on average (Fig. 7C, top row). Inspection of t-SNE plots shows that combinations yielding better performance display clearer separation of cells from different mixture groups (scruff-scran-scran_walktrap), whereas method combinations that perform relatively worse have clusters made up of cells from multiple mixture groups (Additional file 3: Figure S13C-D).

For the cell line datasets, clustering is a much easier task since these comprise relatively fewer (3 or 5) well-separated clusters. On these data, nearly all method combinations obtained ARIs of 1 (Additional file 3: Figure S14A) and both ECA and ECP of 0 (Additional file 3: Figure S14B), except for SC3 with SVM.

T-SNE plots of clustering results returned by different methods for the 3 cell line mixture dataset agree with the known cell types present (Additional file 3: Figure S14C). Overall, zUMIs ranked first, with celseq 2 and scPipe ranked equally second across all normalization and clustering method combinations using the linear model coefficients (Fig. 7C, bottom row). Considering the proportion of variance explained, we observed that for the RNA mixture datasets, clustering methods had a greater influence on performance (Fig. 7E left-hand panel), while for the cell line datasets, the choice of normalization method had a greater influence on the results (Fig. 7E right-hand panel). For both types of data, the preprocessing workflow explained the least variation in clustering performance (Fig. 7E).

Clustering performance assessment on 10x datasets The 10x cell line mixture datasets also have a simple structure ( 3 or 5 expected clusters), and the performance of most method combinations, evaluated using ARI values, was 1 (Additional file 3: Figure S15A), with correct clustering of cells observed via t-SNE plots (Additional file 3: Figure S15B). In terms of ARI, RaceID had the lowest values, followed by Seurat (Additional file 3: Figure S15A). Seurat could not be coerced into producing the expected number of clusters, with an additional cluster of cells always retained, (Additional file 3: Figure S15B bottom row). We added up the number of combinations with both ECP and ECA at 0 and found that scPipe and salmon alevin delivered more optimal combinations, followed by $z U M I s$, indicating that these methods consistently delivered reliable results (Additional file 3: Figure S15C). On average, alevin-fry and Cell Ranger rank among the top 3 in terms of performance as estimated by the coefficients from the linear modeling (Fig. 7D top row).

For the lung tissue datasets, clustering results displayed similar performance, with comparable ARIs (Additional file 3: Figure S16A). In terms of top 5 combinations of entropy, kallisto bustools and salmon alevin delivered results with better performance compared to other workflows (Additional file 3: Figure S16B). Among these combinations, SC3 was the most favored clustering method, followed by scran with the algorithm of fastgreedy across workflows. Overall, kallisto bustools and Optimus rank among the top 2 for performance as assessed by the coefficients from the linear model analysis (Fig. 7D middle row).

For the PBMC datasets, the clustering results were similar across preprocessing workflows when summarized by ARI (Fig. 7B and Additional file 3: Figure S17A). Clustering methods varied in performance, with SC3 performing best, followed by scran with the 
algorithm of walktrap, while RaceID and SC3 with SVM performed relatively worse (Additional file 3: Figure S17A-B). T-SNE plots agreed with ARI values, where combinations with higher values display clearer separation between known cell types, and clustering results that were concordant with the cell labels (Additional file 3: Figure S17C). In terms of the top 5 combinations of entropy, scPipe delivered results with better performance compared to other workflows (Additional file 3: Figure S17D), while results of zUMIs and salmon alevin were evaluated to have higher entropy. Among these combinations, instead of SC3, scran with the algorithm of louvain was the most favored clustering method across workflows. Overall, Cell Ranger and scPipe ranked among the top 2 for performance as assessed by the coefficients from the linear model analysis (Fig. 7D bottom row).

Consistent with observations from the plate-based datasets, the proportion of variation in performance explained in the cell line datasets, lung tissue datasets, and PBMC datasets was greatest for the clustering method while the variation in performance explained by preprocessing and normalization methods were both minimal (Fig. 7E right-hand panel).

\section{Discussion}

\section{Summary of performance of preprocessing workflows}

We compared the performance of 10 preprocessing workflows across CEL-Seq2 and 10x Chromium platforms on datasets with varying biological complexity and explored their quantification characteristics and impact on downstream analysis. In terms of preprocessing workflows designed for CEL-Seq2, the methods compared showed high concordance in quantification, with small discrepancies on detected features. Among them, celseq 2 is more sensitive and returned more non-protein-coding genes. Very similar results were returned by scPipe and scruff which share a lot of the same preprocessing choices, although more biological noise was observed in lowly expressed genes quantified by scPipe due to its UMI quantification approach. scPipe only collapses UMIs that differ by a hamming distance of 1 with more than a 2 -fold difference in counts, which means the UMI counts obtained by scPipe tend to be relatively larger. In terms of downstream analysis, on the simpler (cell line) datasets, nearly all workflows produced clustering results that agreed well with the known cell type labels irrespective of the choice of normalization and clustering methods. On the more complex RNA mixture dataset, with clusters that are less well separated due to the gradient of mixture proportions used in this design, scruff and celseq 2 performed better (Fig. 7C). Our analysis revealed that kallisto bustools (v0.39.3) was unsuitable for use on CEL-Seq2 data due to its UMI handling strategy, which limits the dynamic range that can be observed in the presence of short UMIs (6bp in the case of these CEL-Seq2 datasets). This approach does not cause any noticeable feature detection issues in protocols that use longer UMIs such as 10x Chromium (10-12bp).

For the workflows compared on the 10x Chromium platform, several differences were observed with respect to the detection and quantification of genes. Cell Ranger, together with salmon alevin returned more features with higher abundance, which were mostly comprised of protein coding genes, whereas other workflows recovered more features at the second lower abundance feature peak. kallisto bustools delivered the most distinct count distribution with more features with relatively lower counts (around 100) or in the mid expression range (around 1000). 
Also, we found that alevin-fry, scPipe, and kallisto bustools on the tissue datasets and alevin-fry, scPipe, and zUMIs on the PBMC datasets retained more biological noise, particularly among lowly expressed features compared to other workflows examined. Many of the lowly expressed features were not common between preprocessing workflows. Taken together, these differences indicate the uncertainty in detecting and quantifying lowly expressed features.

Previous studies have illustrated Cell Ranger's bias for genes with low uniqueness [24] and the adverse effect of running it with a full annotation file [32]. Cell Ranger's use of a targeted reference annotation that focuses on protein coding genes and lncRNAs and excludes biotypes that are more difficult to resolve with short-read sequencing, such as small RNAs and pseudogenes, would likely benefit other preprocessing workflows. The use of a modified feature set can not only alleviate multi-mapping issues but also avoid the workflow-specific gene quantification impacts seen in other gene biotypes that are not of principal interest (such as pseudogenes), although this approach would obviously not suit studies in which quantification of such features are of particular interest. We observed that biological signals present in the raw counts were nearly identical between Cell Ranger and the other preprocessing workflows compared. Additionally, after downstream analysis, we observed Cell Ranger's performance to be consistently high and relatively stable, which supports the results of another recent study [32]. Some preprocessing workflows were found to be relatively more or less likely to assign counts to a particular class of genomic features than others. For example, scPipe assigned systematically more reads to pseudogenes, whereas dropSeqPipe, salmon alevin, alevin-fry, and kallisto bustools assigned many fewer reads to this feature class. In addition, for the workflows that use pseudoalignment or selective alignment, kallisto bustools provided more pseudogenes yielding a limited number of counts; alevin-fry and salmon alevin recovered more biological noise in pseudogenes.

After normalization, evaluating the performance of preprocessing workflows based on known biological information uncovered similar performance in all but a few method combinations, indicating high concordance between preprocessing workflows.

When selecting highly variable genes, interestingly, we found that features of different biotypes were present in varying proportions across workflows, with the choice of normalization method also influencing the proportions of different gene biotypes that made it into the HVG list.

Regarding clustering results, similar performance was observed across different workflows and methods on selected datasets. Clustering results that were highly consistent with the provided cell labels were observed on the cell line datasets, and on the lung tissue and PBMC datasets, where cellular complexity was higher, we still observed good performance across all workflows.

Overall, Cell Ranger, alevin-fry performed slightly better on droplet-based datasets on average. Although slightly lower correlations were observed between zUMIs and other workflows, and it uniquely considers intron reads by default, we did not observe improved clustering results by applying it.

Intron reads are indicated to be informative as they likely originate from nascent mRNA [67] and were shown to assist in extracting more information when included in quantification [17]. However, the scRNA-seq data we analyzed both rely on poly(A) selection, which may limit the amount of intron signal that can be extracted. A summary of the advantages 
and limitations of the preprocessing workflows evaluated is presented in Additional file 4: Table S3.

\section{Limitations}

Our benchmarking study is subject to several limitations. First, although there are other protocols such as InDrops, Drop-seq, and Sort-seq, and different protocols are known to influence downstream analysis $[26,68]$, our results are restricted to datasets from two protocols, CEL-Seq2 and 10x Chromium. Second, we did not expand our analysis to include other important aspects of the single-cell data processing, such as dimensionality reduction and feature selection [53]. Extending our benchmarking to cover additional protocols, new preprocessing methods, and other data analysis tasks is left as future work.

Furthermore, the sample types studied are limited to cells from cancer cell lines, primary lung tissue, and PBMCs. Further work could explore the performance of preprocessing workflows on datasets that include a more diverse range of cell types, e.g., cells that make up the tumor microenvironment, although we would anticipate broadly similar results.

Another aspect not investigated in our study is which specific step within different preprocessing workflows has the most influence on performance. Although we observed quantification differences between workflows, we did not delve further into the individual steps within a workflow, which include alignment, deduplication, etc. Previous studies have already provided relatively comprehensive comparisons of alignment and quantification methods $[27,40]$. Hence, the results from our benchmarking study are targeted more to workflow users rather than workflow developers.

\section{Conclusions}

Our assessment investigated the quantification performance of preprocessing workflows and their impact on downstream analysis. We found that scRNA-seq preprocessing workflows varied in their detection and quantification of lowly expressed genes across datasets. However, after subsequent downstream analysis by well-performing normalization and clustering methods, even if the proportion of different gene biotypes detected differed and workflow-specific genes were identified within the various sets of highly variable genes, nearly all combinations delivered good performance with relatively minor differences in the final cell clustering results. Our detailed analysis of 3870 datasets $\times$ method combinations, made possible by the CellBench evaluation framework, finds that the choice of preprocessing workflow has relatively less impact on the results of a single-cell analysis than subsequent downstream analysis steps such as normalization and clustering.

\section{Methods}

\section{Preprocessing workflows compared}

We evaluated 10 publicly available preprocessing workflows in total (Additional file 1: Table S1). Workflows that started from raw FASTQ files and provide a cell-by-gene count matrix as output was chosen. All workflows were installed and run locally, except for Optimus, which was run on Terra (https://app.terra.bio).

For all analyses, the genome, transcriptome (both cDNA and ncRNA), and GTF versions used for human datasets was Ensembl GRCh38, release 98 and for mouse 
datasets, Ensembl GRCm38, release 99. The software versions used were as follows: Cell Ranger (v6.0.0), celseq2 (v0.5.3.3), dropSeqPipe (v0.4.1) (YouTube tutorial link https:// www.youtube.com/watch?v=4bt-azBO-18), kallisto (v0.46.0), bustools (v0.39.3), Optimus (v4.3.2 on Terra), salmon (v1.5.2 for selective alignment), salmon (v1.5.1) and alevin-fry (v0.4.0) contained in the usefulaf (https://github.com/COMBINE-lab/usefulaf) repository, scPipe (v1.8.0), scruff (v1.4.2), zUMIs (v2.5.5 on plate-based datasets and v2.9.7 on droplet-based datasets), bowtie2 (v2.3.4.1), samtools (v1.9), STAR (v2.6.1c) and Rsubread (v2.0.0 for plate-based datasets and v2.4.3 for droplet-based datasets). Parameters within each preprocessing workflow were selected as recommended in the user guides. More details of each preprocessing workflow for each dataset are available at https://github. com/YOU-k/preprocess.

To compare computational performance, we created subsets of the data of varying sizes ranging from $8 \mathrm{M}$ to $100 \mathrm{M}$ for plate-based datasets and $75 \mathrm{M}$ to $600 \mathrm{M}$ for droplet-based datasets. and carried out each analysis on a high-performance cluster (Intel(R) Xeon(R) CPU E5-2690 v4@2.60GHz). Maximum memory at 200GB and the time limit at $48 \mathrm{~h}$ were set for each submission. We required 1 node and 8 PPNs for each run and claimed 8 cores in the scripts if there were parameters that allowed this; if not, 8 threads were claimed. We ran each workflow three times on each dataset and calculated the mean and standard deviation of the performance measures, which include CPU utilization, memory usage, and run time. To measure their parallel scaling performance, threads were set as 4 , $8,12,16,32$, and run times were recorded.

\section{Datasets}

We selected 11 public datasets and created 1 new dataset for benchmarking (Additional file 2: Table S2). The scmixology datasets are available from GEO under accession number GSE118767 [5]. We selected plate-based CEL-Seq2 and 10x v2 droplet-based datasets containing cells from human lung adenocarcinoma cell lines that involved "pseudo-cells" created by mixing cells ( 3 datasets) or bulk RNA mixtures (1 dataset) in different combinations using CEL-seq 2 or actual single cells ( 1 dataset with 3 cell lines and 1 dataset with 5 cell lines). Annotation files for CBs with cell types were available at https://github.com/ LuyiTian/sc_mixology.

The new dataset was created using single cells from the same five human lung adenocarcinoma cell lines (HCC827, H1975, A549, H838, and H2228) that were cultured separately. Cells were counted using Chamber Slides, and roughly 2 million cells from each cell line were mixed and processed by the 10x Chromium single cell platform using v3 chemistry. Afterward, libraries were sequenced on an Illumina Nextseq 500. Raw data from this experiment are available from GEO under accession number GSE154870. To generate its annotation files, demuxlet ( https:/github.com/statgen/demuxlet) was used to deconvolve cell identity via genetic information using the intermediate bam file obtained from the scPipe workflow.

Another dataset profiled mouse lung tissue from the Tabula Muris study, available under GEO under accession number GSE109774 [41]. Raw bam files from channel 10X_P7_8 and 10X_P7_9 were downloaded and converted to raw FASTQ files by bamtofastq (v1.2.0) (https://support.10xgenomics.com/docs/bamtofastq). Annotation files for CBs with cell types were available from http://tabula-muris.ds.czbiohub.org. 
The final datasets that profiled human peripheral blood mononuclear cells (PBMC) were provided by 10x. A 5k and 10k PBMC dataset were downloaded from the 10x website (from https://support.10xgenomics.com/single-cell-gene-expression/datasets/3.0.2/5k pbmc_v3 and https://support.10xgenomics.com/single-cell-gene-expression/datasets/3. 0.0/pbmc_10k_v3). They were both sequenced with the v3 chemistry of the Chromium $10 x$ system. To generate cell type annotation files on each dataset, we followed the online Bioconductor tutorial [69] as follows:

- Quality control of cells was performed using scater (v1.14.6) whereby cells with total read counts or number of genes detected greater than 3 median absolute deviations (MADs) below the median (both calculated on the $\log _{1} 0$-scale) or cells where the percentage of mitochondrial counts was 3 MADs above the median were removed from the filtered matrices downloaded from the 10x website.

- scran was used to normalize the data, and the top $2 \mathrm{k}$ highly variable genes were selected with modelGeneVar and getTopHVGs.

- The shared nearest-neighbor (SNN) graph was built using the top 20 PCs, and cluster_louvain was applied to cluster cells.

- A UMAP was generated on the first 20 PCs to embed the datasets into two dimensions for visualization.

- scDblFinder: : findDoubletclusters (v1.4.0) was used to find clusters of doublets. Cells from doublet clusters were removed and the analysis steps after quality control were rerun.

- To assign cell types to clusters, we used canonical marker genes (Additional file 3: Figure S18). The BlueprintEncodeData [70] were also used as reference to annotate cells with the usage of SingleR (v1.4.1) [71].

The long-read Nanopore single-cell datasets used the same five human lung adenocarcinoma cell line mixture samples processed by the 10x Chromium single cell runs using v2 and v3 chemistry according to the protocols described in Tian et al. [49]. The longread based count-matrices provided by the authors were used to compare the abundance of particular gene biotypes with the matching short-read data.

\section{Cell quality control}

In an attempt to standardize cell filtering, we applied isOutlier from the scater package (v1.14.6) setting library size, the number of detected features, and percentage of mitochondrial genes per cell as filtering indicators with nmads $=3$ for all cell-by-gene count matrices created by different preprocessing workflows. Additionally, we applied emptyDrops from the DropletUtils package (v1.6.1) [14] (as recommended in [69]) to all selected 10x datasets. For the lung tissue dataset and 10xv2_3cell-line dataset, emptyDrops failed to generate a reasonable number of cells (i.e. around 60 thousand cells in each on 10xv2_3cell-line for zUMIs and kallisto bustools, which is well above expectation of around 3k cells for other workflows), so we used Cell Ranger v2 filtering to distinguish true cells on these datasets. salmon alevin still provided relatively more cells on the 10xv2_lung-tissue1 datasets, and as most of these cells did not have a cell type label (Additional file 3: Figure S3C right-most panel). Therefore, we restricted our analysis to only include cells with labels on this dataset across workflows. Values of quality metrics after filtering are provided in Additional file 5: Table S4. 
The number of detected genes per gene biotypes, total counts per gene, total counts per cell, and Pearson's correlation were all calculated based on the cell-by-gene count matrices obtained before filtering by scater. Here, the correlation was calculated using common cells and common features detected across all preprocessing workflows. GLMPCs were calculated using the GLMPCA package (v0.2.0) and UpSetR (v1.4.0) plots of cells found in common between preprocessing methods after filtering with using scater were generated for different datasets.

Because $\mathrm{CB}$ annotation files were generated based on one specific workflow, and the original analyses adopted different filtering strategies, it was possible to recover cells regarded as good quality by some preprocessing workflows that were not listed in the CB annotation files. Such cells were retained for further analysis and only removed upon calculating tailored evaluation metrics that required cell labels. Doublets detected by demuxlet in both the CEL-Seq2 and 10x cell line datasets were removed before normalization.

\section{BCV plot and biological signal on raw counts}

For biological noise and signals, genes with specific biotypes were firstly extracted. Next, the biological coefficient of variation $(B C V)$ was calculated using the filtered raw count matrix using the edgeR: : estimateDispersion function (v3.28.1) [72] and trended.dispersion was plotted using a loess line or directly with points. In the $\mathrm{BCV}$ plots, the $\mathrm{x}$-axis displays the log-transformed counts obtained after scran normalization. Silhouette widths were calculated using the first 2 GLMPCs for cell line datasets and the first 20 GLMPCs for lung tissue and PBMC datasets.

For clustering of $n$ observations (here a cluster refers to a specified group of cells), the silhouette width of observation $i$ is defined as:

$$
\operatorname{sil}(i)=\frac{b(i)-a(i)}{\max (a(i), b(i))} \in[-1,1]
$$

where $a(i)$ represents the average (here Euclidean distance based on the top selected PCs) dissimilarity between the $i$ th cell and all other cells in the cluster where $i$ belongs to. Here, $b(i)$ is calculated as

$$
b(i)=\min _{C} d(i, C)
$$

where $d(i, C)$ represents the average dissimilarity of $i$ to all observations in other clusters $C$. The cluster: : silhouette (v2.1.2) function [73] was used to calculate silhouette width.

To generate t_SNE plots, the same sets of genes across biotypes were extracted after normalized by scran. PCA was performed on them separately. The First 2 PCs on cell line datasets and the first 20 PCs on the lung tissue and PBMC datasets were used to create a t_SNE visualization.

\section{Data normalization}

Five normalization methods were used to explore the impact the choice of preprocessing workflow has on this step and subsequent downstream analysis. The baseline no normalization option refers to the analysis of the raw counts directly without any further processing (this option was not used in downstream analysis). DESeq2 (v1.26.0), Linnorm (v2.10.0) and scran (v1.14.6) were used with default settings. For scone, we set 
the maximum number of RUVg factors and maximum number of quality PCs both as 0 . For sctransform (v0.3.2) both the default method and the method that uses glmGamPoi (v1.2.0) [59] were applied with 1500 features specified as the number of variable features after ranking by residual variance.

To evaluate, we performed PCA with normalized counts firstly with default settings in scater. Next, silhouette widths were calculated with the first 2 PCs according to known cell clusters (provided by cell line identity or mixture proportion information) for all datasets except the tissue and PBMC datasets. Considering the biological complexity of tissue and PBMC datasets, silhouette widths were calculated using the first 20 PCs. Higher silhouette widths indicated better preservation of biological signals. Additionally, variance explained by library sizes and known cell groups on the first five PCs were summed up respectively as unwanted variation and wanted variation to assess whether known biological variation was preserved and confounded technical effects were well handled on all plate-based datasets and droplet-based cell line datasets. For the tissue and PBMC datasets, variance explained from the first 20 PCs were partitioned into wanted and unwanted variation and then summed up.

\section{Clustering}

Results from normalization were not directly applied with clustering methods. Except for sctransform, normalized counts were already selected with top $1.5 \mathrm{k}$ highly variable genes (HVGs), top 1.5k HVGs were obtained with scran : : modelGeneVar and scran: : getTopHVGs. Clustering methods from mainly four packages, SC3 (v1.14.0), Seurat (v3.1.3), RaceID (v0.1.7), and scran with igraph (v1.2.5), were used. To make it easier to interpret, we provided the number of clusters or specified related parameters with a range of values to reach the true value of the number of clusters. Other parameters were specified based on either the default settings or the author's guidance from the user manual.

For SC3, both the classic unsupervised method and combined Support Vector Machine (SVM) method were used. RaceID parameters as suggested in the user reference manual were chosen. The required number of clusters were directly provided to SC3 and RaceID. For scran, the number of nearest neighbors was specified at 5, 10, 30, 50 and 100 to build a SNN graph. Algorithms fast greedy, Louvain, and walktrap in igraph were applied afterwards. For Seurat, we clustered specifying resolution at 0 . 2, 0 . 4, 0 . 6, 0 . 8, 1.0, 1.2, 1.4 with the algorithms of Louvain and SLM. With RaceID and SC3, the desired number of clusters can be provided directly as a parameter. When applying scran and Seurat, the clustering solution that returned either the expected number of clusters or the closest number to that were taken as the optimal solution for benchmarking.

To evaluate clustering performance, the entropy of cluster accuracy (ECA), entropy of purity (ECP) [5] and adjusted Rand index (ARI) was used to assess intra-cluster similarity, external criterion, and similarity with known clusters, respectively. ECA and ECP are defined as:

$$
E C A=-\frac{\sum_{i=1}^{M} \sum_{j=1}^{N_{i}} p\left(x_{j}\right) \log \left(p\left(x_{j}\right)\right)}{M}
$$

where $M$ denotes the number of clusters generated from a method (the clustering solution to be evaluated) and $N_{i}$ denotes in $i$ th cluster based on the ground truth (here the provided 
labels), and

$$
E C P=-\frac{\sum_{i=1}^{N} \sum_{j=1}^{M_{i}} p\left(x_{j}\right) \log \left(p\left(x_{j}\right)\right)}{N}
$$

where $N$ denotes the number of clusters from the ground truth and $M_{i}$ denotes in $i$ th cluster based on the generated clusters. ECA measures the diversity of known cell groups within each cluster provided by given methods, and ECP measures the diversity of the cluster labels within each of the known groups. Low values of ECA and ECP are favorable. The mclust : : adjustedRandIndex (v5.4.7) function was used to calculate ARI.

To summarize the results of each analysis, we performed ANOVA with the following model: metric $\sim$ preprocess_workflow + norm_method + cluster_method + design.

We also fitted a linear model using the $1 \mathrm{~m}$ function with the listed metrics as the dependent variable and the experimental designs and specific methods as covariates. The coefficient obtained for each method indicated to what extent these methods were influencing the clustering performance. Then, the average weighted rank of coefficients on preprocessing workflows across three metrics (ARI, ECA, and ECP) are calculated as a summary. Example t-SNE plots (created using scater) allowed visual assessment of different combinations' performance. The rest of the figures were created using ggplot2 (v3.3.0) and heatmaps were created using pheatmap (v1.0.12).

\section{Benchmarking pipelines}

CellBench (v1.2.0) was used to compare different methods as modules. The preprocessing workflows were individually applied to each dataset and the resulting cell-by-gene count matrix were input to CellBench: :apply_methods () [39].

\section{Supplementary Information}

The online version contains supplementary material available at https://doi.org/10.1186/s13059-021-02552-3.

Additional file 1: Table S1: Summary of the scRNA-seq preprocessing workflows compared.

Additional file 2: Table S2: Summary of the benchmarking datasets analyzed.

Information of experimental designs, GEO accession numbers, data structure, biological noise levels, and expected number of cells and number of clusters are provided.

Additional file 3: Supplementary Figures.

Additional file 4: Table S3: Summary of the advantages and limitations of the preprocessing workflows evaluated.

Additional file 5: Table S4: Summary of quality control thresholds across datasets and preprocessing workflows.

Additional file 6: Review history.

\section{Acknowledgements}

This work was supported by funding from the Chan Zuckerberg Initiative DAF, an advised fund of Silicon Valley Community Foundation (Grant Nos. 2018-182819 and 2019-002443 to M.E.R.), Australian National Health and Medical Research Council (NHMRC) Project Grant (1143163 to M.E.R.) and Fellowship (1104924 to M.E.R.), the Australian Research Council (Discovery Project Nos. 180101405 and 200102903 to M.E.R.), the Genomics Innovation Hub, Victorian State Government Operational Infrastructure Support, Australian Government NHMRC IRIISS, and support from the Australian Cancer Research Foundation.

Peer review information

Barbara Cheifet was the primary editor of this article and managed its editorial process and peer review in collaboration with the rest of the editorial team.

Review history

The review history is available as Additional file 6.

\section{Authors' contributions}

Y.Y. conducted data analysis, generated all figures, and wrote the manuscript. L.T., S.S, and X.D. performed the data analysis. L.T. and J.S.J. performed the experiments. M.E.R. designed the study. M.E.R. and P.F.H supervised the analysis and wrote the manuscript. All authors read and approved the final manuscript. 


\section{Availability of data and materials}

The scmixology datasets are available from GEO under accession numbers GSE1 18767 [74] and GSE154870 [75]. The mouse lung tissue data from the Tabula Muris study is available under GEO under accession number GSE109774 [76]. The human PBMC datasets are available from the 10x website (the $5 \mathrm{k}$ dataset is from https://support.10xgenomics.com/ single-cell-gene-expression/datasets/3.0.2/5k_pbmc_v3 and the 10k dataset is from https://support.10xgenomics.com/ single-cell-gene-expression/datasets/3.0.0/pbmc_10k_v3). Code for the preprocessing analysis and wrappers for use in CellBench for the methods compared are available from GitHub at https://github.com/YOU-k/preprocess [77].

\section{Declarations}

\section{Ethics approval and consent to participate}

Not applicable.

\section{Competing interests}

The authors declare that they have no competing interests.

\section{Author details}

${ }^{1}$ Epigenetics and Development Division, The Walter and Eliza Hall Institute of Medical Research, 1G Royal Parade, Parkville, Australia. ${ }^{2}$ Department of Medical Biology, The University of Melbourne, Parkville, Australia. ${ }^{3}$ Australian Genome Research Facility, Victorian Comprehensive Cancer Centre, Melbourne, Australia. ${ }^{4}$ Microbiological Diagnostic Unit Public Health Laboratory, Department of Microbiology and Immunology, The University of Melbourne at The Peter Doherty Institute for Infection and Immunity, Melbourne, Australia. ${ }^{5}$ Single-Cell Open Research Endeavour (SCORE), The Walter and Eliza Hall Institute of Medical Research, 1G Royal Parade, Parkville, Australia. ${ }^{6}$ School of Mathematics and Statistics, The University of Melbourne, Parkville, Australia.

Received: 17 June 2021 Accepted: 22 November 2021

Published online: 14 December 2021

\section{References}

1. Zappia L, Phipson B, Oshlack A. Exploring the single-cell RNA-seq analysis landscape with the scRNA-tools database. PLoS Comput Biol. 2018;14(6):e1006245. https://doi.org/10.1371/journal.pcbi.1006245.

2. Svensson V, Vento-Tormo R, Teichmann S. Exponential scaling of single-cell RNA-seq in the past decade. Nat Protoc. 2018;13(4):599-604. https://doi.org/10.1038/nprot.2017.149.

3. Duò A, Robinson M, Soneson C. A systematic performance evaluation of clustering methods for single-cell RNA-seq data. F1000Research. 2018;7:1141. https://doi.org/10.12688/f1000research.15666.3.

4. Saelens W, Cannoodt R, Todorov H, Saeys Y. A comparison of single-cell trajectory inference methods. Nat Biotechnol. 2019;37(5):547-54. https://doi.org/10.1038/s41587-019-0071-9.

5. Tian L, Dong X, Freytag S, Lê Cao K, Su S, JalalAbadi A, Amann-Zalcenstein D, Weber T, Seidi A, Jabbari J, Naik S, Ritchie M. Benchmarking single cell RNA-sequencing analysis pipelines using mixture control experiments. Nat Methods. 2019;16(6):479-87. https://doi.org/10.1038/s41592-019-0425-8.

6. Abdelaal T, Michielsen L, Cats D, Hoogduin D, Mei H, Reinders M, Mahfouz A. A comparison of automatic cell identification methods for single-cell RNA sequencing data. Genome Biol. 2019;20(1):194. https://doi.org/10.1186/ s13059-019-1795-Z

7. Conesa A, Madrigal P, Tarazona S, Gomez-Cabrero D, Cervera A, McPherson A, Szcześniak M, Gaffney D, Elo L, Zhang X, Mortazavi A. A survey of best practices for RNA-seq data analysis. Genome Biol. 2016;17:13. https://doi.org/ 10.1186/s13059-016-0881-8.

8. Luecken M, Theis F. Current best practices in single-cell RNA-seq analysis: a tutorial. Mol Syst Biol. 2019;15(6):e8746. https://doi.org/10.15252/msb.20188746.

9. Kivioja T, Vähärautio A, Karlsson K, Bonke M, Enge M, Linnarsson S, Taipale J. Counting absolute numbers of molecules using unique molecular identifiers. Nat Methods. 2012;9(1):72-4. https://doi.org/10.1038/nmeth.1778.

10. Islam S, Zeisel A, Joost S, La Manno G, Zajac P, Kasper M, Lönnerberg P, Linnarsson S. Quantitative single-cell RNA-seq with unique molecular identifiers. Nat Methods. 2014;11(2):163-6. https://doi.org/10.1038/nmeth.2772.

11. Hashimshony T, Wagner F, Sher N, Yanai I. CEL-Seq: Single-Cell RNA-Seq by Multiplexed Linear Amplification. Cell Rep. 2012;2(3):666-73. https://doi.org/10.1016/j.celrep.2012.08.003.

12. Zheng G, Terry J, Belgrader P, Ryvkin P, Bent Z, Wilson R, Ziraldo S, Wheeler T, McDermott G, Zhu J, Gregory M, Shuga J, Montesclaros L, Underwood J, Masquelier D, Nishimura S, Schnall-Levin M, Wyatt P, Hindson C, Bharadwaj R, Wong A, Ness K, Beppu L, Deeg H, McFarland C, Loeb K, Valente W, Ericson N, Stevens E, Radich J, Mikkelsen T, Hindson B, Bielas J. Massively parallel digital transcriptional profiling of single cells. Nat Commun. 2017;8:14049. https://doi.org/10.1038/ncomms14049.

13. Klein A, Mazutis L, Akartuna I, Tallapragada N, Veres A, Li V, Peshkin L, Weitz D, Kirschner M. Droplet barcoding for single-cell transcriptomics applied to embryonic stem cells. Cell. 2015;161(5):1187-201. https://doi.org/10.1016/j cell.2015.04.044.

14. Lun A, Riesenfeld S, Andrews T, Dao T, Gomes T, Marioni J. EmptyDrops: Distinguishing cells from empty droplets in droplet-based single-cell RNA sequencing data. Genome Biol. 2019;20(1):63. https://doi.org/10.1186/s13059-0191662-y.

15. Smith T, Heger A, Sudbery I. UMI-tools: Modeling sequencing errors in Unique Molecular Identifiers to improve quantification accuracy. Genome Res. 2017;27(3):491-9. https://doi.org/10.1101/gr.209601.116.

16. Tian L, Su S, Dong X, Amann-Zalcenstein D, Biben C, Seidi A, Hilton D, Naik S, Ritchie M. scPipe: a flexible R/Bioconductor preprocessing pipeline for single-cell RNA-sequencing data. PLoS Comput Biol. 2018;14(8): e1006361. https://doi.org/10.1371/journal.pcbi.1006361. 
17. Parekh S, Ziegenhain C, Vieth B, Enard W, Hellmann I. zUMls - A fast and flexible pipeline to process RNA sequencing data with UMls. GigaScience. 2018;7(6):giy059. https://doi.org/10.1093/gigascience/giy059.

18. Bray N, Pimentel H, Melsted P, Pachter L. Near-optimal probabilistic RNA-seq quantification. Nat Biotechnol. 2016;34(5):525-7. https://doi.org/10.1038/nbt.3519.

19. Patro R, Duggal G, Love M, Irizarry R, Kingsford C. Salmon provides fast and bias-aware quantification of transcript expression. Nat Methods. 2017;14(4):417-9. https://doi.org/10.1038/nmeth.4197.

20. Regev A, Teichmann S, Lander E, Amit I, Benoist C, Birney E, Bodenmiller B, Campbell P, Carninci P, Clatworthy M, Clevers H, Deplancke B, Dunham I, Eberwine J, Eils R, Enard W, Farmer A, Fugger L, Göttgens B, Hacohen N, Haniffa M, Hemberg M, Kim S, Klenerman P, Kriegstein A, Lein E, Linnarsson S, Lundberg E, Lundeberg J, Majumder P, Marioni J, Merad M, Mhlanga M, Nawijn M, Netea M, Nolan G, Pe'er D, Phillipakis A, Ponting C, Quake S, ReikW, Rozenblatt-Rosen O, Sanes J, Satija R, Schumacher T, Shalek A, Shapiro E, Sharma P, Shin J, Stegle O, Stratton M, Stubbington M, Theis F, Uhlen M, Van Oudenaarden A, Wagner A, Watt F, Weissman J, Wold B, Xavier R, Yosef N. The human cell atlas. eLife. 2017;6:e27041. https://doi.org/10.7554/eLife.27041.

21. Human Cell Atlas skylab. Optimus V1.3.6 and V2.0.0. https://github.com/HumanCellAtlas/skylab/tree/master/ pipelines/optimus.

22. Papatheodorou I, Moreno P, Manning J, Fuentes A, George N, Fexova S, Fonseca N, Füllgrabe A, Green M, Huang $\mathrm{N}$, Huerta L, Iqbal H, Jianu M, Mohammed S, Zhao L, Jarnuczak A, Jupp S, Marioni J, Meyer K, Petryszak R, Prada Medina C, Talavera-López C, Teichmann S, Vizcaino J, Brazma A. Expression Atlas update: From tissues to single cells. Nucleic Acids Res. 2020;48(D1):77-83. https://doi.org/10.1093/nar/gkz947.

23. Melsted P, Sina Booeshagh A, Liu L, Gao F, Lu L, Min K, da Veiga Beltrame E, Hjörleifsson K, Gehring J, Pachter L. Modular, efficient and constant-memory single-cell RNA-seq preprocessing. Nat Biotechnol. 2021;39(7):813-8. https://doi.org/10.1038/s41587-021-00870-2.

24. Srivastava A, Malik L, Smith T, Sudbery I, Patro R. Alevin efficiently estimates accurate gene abundances from dscRNA-seq data. Genome Biol. 2019;20(1):65. https://doi.org/10.1186/s13059-019-1670-y.

25. Petukhov V, Guo J, Baryawno N, Severe N, Scadden D, Samsonova M, Kharchenko P. dropEst: Pipeline for accurate estimation of molecular counts in droplet-based single-cell RNA-seq experiments. Genome Biol. 2018;19(1):78. https://doi.org/10.1186/s13059-018-1449-6.

26. Chen W, Zhao Y, Chen X, Yang Z, XuX, Bi Y, Chen V, Li J, Choi H, Ernest B, Tran B, Mehta M, Kumar P, Farmer A, Mir A, Mehra U, Li J, Moos M, Xiao W, Wang C. A multicenter study benchmarking single-cell RNA sequencing technologies using reference samples. Nat Biotechnol. 2021;39(9):1103-14. https://doi.org/10.1038/s41587-02000748-9.

27. Vieth B, Parekh S, Ziegenhain C, Enard W, Hellmann I. A systematic evaluation of single cell RNA-seq analysis pipelines. Nat Commun. 2019;10(1):4667. https://doi.org/10.1038/s41467-019-12266-7.

28. Gao M, Ling M, Tang X, Wang S, Xiao X, Qiao Y, Yang W, Yu R. Comparison of high-throughput single-cell RNA sequencing data processing pipelines. Brief Bioinform. 2021;22(3):bbaa116. https://doi.org/10.1093/bib/bbaa1 16.

29. Zakeri M, Srivastava A, Sarkar H, Patro R. A like-for-like comparison of lightweight-mapping pipelines for single-cell RNA-seq data pre-processing. bioRxiv. 2021. https://doi.org/10.1101/2021.02.10.430656.

30. Booeshaghi A, Pachter L. Benchmarking of lightweight-mapping based single-cell RNA-seq pre-processing. bioRxiv. 2021. https://doi.org/10.1101/2021.01.25.428188.

31. He D, Zakeri M, Sarkar H, Soneson C, Srivastava A, Patro R. Alevin-fry unlocks rapid, accurate, and memory-frugal quantification of single-cell RNA-seq data. bioRxiv. 2021. https://doi.org/10.1101/2021.06.29.450377.

32. Schulze Brüning R, Tombor L, Schulz M, Dimmeler S, John D. Comparative analysis of common alignment tools for single cell RNA sequencing. bioRxiv. 2021. https://doi.org/10.1101/2021.02.15.430948.

33. Kaminow B, Yunusov D, Dobin A. STARsolo: accurate, fast and versatile mapping/quantification of single-cell and single-nucleus RNA-seq data. bioRxiv. 2021. https://doi.org/10.1101/2021.05.05.442755.

34. Soneson C, Srivastava A, Patro R, Stadler M. Preprocessing choices affect RNA velocity results for droplet scRNA-seq data. PLoS Comput Biol. 2021;17(1):e1008585. https://doi.org/10.1371/journal.pcbi.1008585.

35. Hashimshony T, Senderovich N, Avital G, Klochendler A, de Leeuw Y, Anavy L, Gennert D, Li S, Livak K, Rozenblatt-Rosen O, Dor Y, Regev A, Yanai I. CEL-Seq2: Sensitive highly-multiplexed single-cell RNA-Seq. Genome Biol. 2016;17:77. https://doi.org/10.1186/s13059-016-0938-8.

36. Roelli P, Mueller S, Girardo C. dropSeqPipe V0.4.1. https://github.com/Hoohm/dropSeqPipe.

37. Wang Z, Hu J, Johnson W, Campbell J. Scruff: An R/Bioconductor package for preprocessing single-cell RNA-sequencing data. BMC Bioinformatics. 2019;20(1):222. https://doi.org/10.1186/s12859-019-2797-2.

38. Ramsköld D, Luo S, Wang Y, Li R, Deng Q, Faridani O, Daniels G, Khrebtukova I, Loring J, Laurent L, Schroth G, Sandberg R. Full-length mRNA-Seq from single-cell levels of RNA and individual circulating tumor cells. Nat Biotechnol. 2012;30(8):777-82. https://doi.org/10.1038/nbt.2282.

39. Su S, Tian L, Dong X, Hickey P, Freytag S, Ritchie M. CellBench: R/Bioconductor software for comparing single-cell RNA-seq analysis methods. Bioinformatics. 2020;36(7):2288-90. https://doi.org/10.1093/bioinformatics/btz889.

40. Srivastava A, Malik L, Sarkar H, Zakeri M, Almodaresi F, Soneson C, Love M, Kingsford C, Patro R. Alignment and mapping methodology influence transcript abundance estimation. Genome Biol. 2020;21(1):239. https://doi.org/10. 1186/s13059-020-02151-8.

41. Tabula Muris Consortium. Single-cell transcriptomics of 20 mouse organs creates a Tabula Muris. Nature. 2018;562(7727):367-72. https://doi.org/10.1038/s41586-018-0590-4.

42. Ahlmann-Eltze C, Huber W. glmGamPoi: Fitting Gamma-Poisson generalized linear models on single cell count data. Bioinformatics. 2020;36(24):5701-2. https://doi.org/10.1093/bioinformatics/btaa1009.

43. Townes F, Hicks S, Aryee M, Irizarry R. Feature selection and dimension reduction for single-cell RNA-Seq based on a multinomial model. Genome Biol. 2019;20(1):295. https://doi.org/10.1186/s13059-019-1861-6.

44. Holik A, Law C, Liu R, Wang Z, Wang W, Ahn J, Asselin-Labat M, Smyth G, Ritchie M. RNA-seq mixology: Designing realistic control experiments to compare protocols and analysis methods. Nucleic Acids Res. 2017;45(5): e30. https://doi.org/10.1093/nar/gkw1063.

45. Deschamps-Francoeur G, Simoneau J, Scott M. Handling multi-mapped reads in RNA-seq. Comput Struct Biotechnol J. 2020;18:1569-76. https://doi.org/10.1016/j.csbj.2020.06.014. 
46. Everaert C, Luypaert M, Maag J, Cheng Q, DInger M, Hellemans J, Mestdagh P. Benchmarking of RNA-sequencing analysis workflows using whole-transcriptome RT-qPCR expression data. Sci Rep. 2017;7(1):1559. https://doi.org/10. 1038/s41598-017-01617-3.

47. Wu D, Yao J, Ho K, Lambowitz A, Wilke C. Limitations of alignment-free tools in total RNA-seq quantification. BMC Genom. 2018;19(1):510. https://doi.org/10.1186/s12864-018-4869-5.

48. McCarthy D, Chen Y, Smyth G. Differential expression analysis of multifactor RNA-Seq experiments with respect to biological variation. Nucleic Acids Res. 2012;40(10):4288-97. https://doi.org/10.1093/nar/gks042.

49. Tian L, Jabbari J, Thijssen R, Gouil Q, Amarasinghe S, Voogd O, Kariyawasam H, Du M, Schuster J, Wang C, et al. Comprehensive characterization of single-cell full-length isoforms in human and mouse with long-read sequencing. Genome Biol. 2021;22(1):310. https://doi.org/10.1186/s13059-021-02525-6.

50. Troskie R, Jafrani Y, Mercer T, Ewing A, Faulkner G, Cheetham S. Long-read cDNA sequencing identifies functional pseudogenes in the human transcriptome. Genome Biol. 2021;22(1):146. https://doi.org/10.1186/s13059-02102369-0.

51. Mantere T, Kersten S, Hoischen A. Long-read sequencing emerging in medical genetics. 2019. https://doi.org/10. 3389/fgene.2019.00426.

52. Zheng H, Brennan K, Hernaez M, Gevaert O. Benchmark of long non-coding RNA quantification for RNA sequencing of cancer samples. GigaScience. 2019;8(12):giz145. https://doi.org/10.1093/gigascience/giz145.

53. Germain P, Sonrel A, Robinson M. PipeComp, a general framework for the evaluation of computational pipelines, reveals performant single cell RNA-seq preprocessing tools. Genome Biol. 2020;21(1):227. https://doi.org/10.1186/ s13059-020-02136-7.

54. Cole M, Risso D, Wagner A, DeTomaso D, Ngai J, Purdom E, Dudoit S, Yosef N. Performance assessment and selection of normalization procedures for single-cell RNA-Seq. Cell Syst. 2019;8(4):315-3288. https://doi.org/10. 1016/j.cels.2019.03.010.

55. Love M, Huber W, Anders S. Moderated estimation of fold change and dispersion for RNA-seq data with DESeq2. Genome Biol. 2014;15(12):550. https://doi.org/10.1186/s13059-014-0550-8.

56. Lun A, Bach K, Marioni J. Pooling across cells to normalize single-cell RNA sequencing data with many zero counts. Genome Biol. 2016;17:75. https://doi.org/10.1186/s13059-016-0947-7.

57. Yip S, Wang P, Kocher J, Sham P, Wang J. Linnorm: improved statistical analysis for single cell RNA-seq expression data. Nucleic Acids Res. 2017;45(22):179. https://doi.org/10.1093/nar/gkx828.

58. Hafemeister C, Satija R. Normalization and variance stabilization of single-cell RNA-seq data using regularized negative binomial regression. Genome Biol. 2019;20(1):296. https://doi.org/10.1186/s13059-019-1874-1.

59. Ahlmann-Eltze C, Huber W. glmgampoi: fitting gamma-poisson generalized linear models on single cell count data. Bioinformatics. 2020;36(24):5701-2.

60. Freytag S, Tian L, Lönnstedt I, Ng M, Bahlo M. Comparison of clustering tools in R for medium-sized 10x Genomics single-cell RNA-sequencing data. F1000Research. 2018;7:1297. https://doi.org/10.12688/f1000research.15809.2.

61. Krzak M, Raykov Y, Boukouvalas A, Cutillo L, Angelini C. Benchmark and Parameter Sensitivity Analysis of Single-Cell RNA Sequencing Clustering Methods. Front Genet. 2019;10:1253. https://doi.org/10.3389/fgene.2019.01253.

62. Herman J, Sagar, Grün D. FatelD infers cell fate bias in multipotent progenitors from single-cell RNA-seq data. Nat Methods. 2018;15(5):379-86. https://doi.org/10.1038/nmeth.4662.

63. Kiselev V, Kirschner K, Schaub M, Andrews T, Yiu A, Chandra T, Natarajan K, Reik W, Barahona M, Green A, Hemberg M. SC3: Consensus clustering of single-cell RNA-seq data. Nat Methods. 2017;14(5):483-6. https://doi.org/ 10.1038/nmeth.4236.

64. Stuart T, Butler A, Hoffman P, Hafemeister C, Papalexi E, Mauck W, Hao Y, Stoeckius M, Smibert P, Satija R. Comprehensive Integration of Single-Cell Data. Cell. 2019;177(7):1888-190221. https://doi.org/10.1016/j.cell.2019. 05.031.

65. Blondel V, Guillaume J, Lambiotte R, Lefebvre E. Fast unfolding of communities in large networks. J Stat Mech Theory Exp. 2008;2008:P10008. http://dx.doi.org/10.1088/1742-5468/2008/10/P10008.

66. Hubert L, Arabie P. Comparing partitions. J Classif. 1985;2(1):193-218. https://doi.org/10.1007/BF01908075.

67. Gaidatzis D, Burger L, Florescu M, Stadler M. Analysis of intronic and exonic reads in RNA-seq data characterizes transcriptional and post-transcriptional regulation. Nat Biotechnol. 2015;33(7):722-9. https://doi.org/10.1038/nbt. 3269.

68. Mereu E, Lafzi A, Moutinho C, Ziegenhain C, McCarthy D, Álvarez-Varela A, Batlle E, Sagar, Grün D, Lau J, Boutet S, Sanada C, Ooi A, Jones R, Kaihara K, Brampton C, Talaga Y, Sasagawa Y, Tanaka K, Hayashi T, Braeuning C, Fischer C, Sauer S, Trefzer T, Conrad C, Adiconis X, Nguyen L, Regev A, Levin J, Parekh S, Janjic A, Wange L, Bagnoli J, Enard W, Gut M, Sandberg R, Nikaido I, Gut I, Stegle O, Heyn H. Benchmarking single-cell RNA-sequencing protocols for cell atlas projects. Nat Biotechnol. 2020;38(6):747-55. https://doi.org/10.1038/s41587-020-0469-4.

69. Amezquita R, Lun A, Becht E, Carey V, Carpp L, Geistlinger L, Marini F, Rue-Albrecht K, Risso D, Soneson C, Waldron L, Pagès H, Smith M, Huber W, Morgan M, Gottardo R, Hicks S. Orchestrating single-cell analysis with Bioconductor. Nat Methods. 2020;17(2):137-45. https://doi.org/10.1038/s41592-019-0654-x.

70. Martens J, Stunnenberg H. Blueprint: mapping human blood cell epigenomes. Haematologica. 2013;98(10):1487-9.

71. Aran D, Looney A, Liu L, Wu E, Fong V, Hsu A, Chak S, Naikawadi R, Wolters P, Abate A, et al. Reference-based analysis of lung single-cell sequencing reveals a transitional profibrotic macrophage. Nat Immunol. 2019;20(2): $163-72$.

72. Robinson M, McCarthy D, Smyth G. edgeR: A Bioconductor package for differential expression analysis of digital gene expression data. Bioinformatics. 2009;26(1):139-40. https://doi.org/10.1093/bioinformatics/btp616.

73. Maechler M, Rousseeuw P, Struyf A, Hubert M, Hornik K, et al. Cluster: cluster analysis basics and extensions. R Packag version. Package version 2.1.2.

74. Tian L, Amann-Zalcenstein D, Weber T, Seidi A, Jabbari J, Naik S, Ritchie M. Designing a Single Cell RNA Sequencing Benchmark Dataset to Compare Protocols and Analysis Methods. https://www.ncbi.nlm.nih.gov/geo/ query/acc.cgi?acc=GSE118767. 
75. Tian L, Jabbari J, Gouil Q, Ritchie M. Long and short-read single cell RNA-seq profiling of human lung adenocarcinoma cell lines using 10XV3 chemistry. https://www.ncbi.nlm.nih.gov/geo/query/acc.cgi?acc= GSE154870.

76. The Tabula Muris Consortium. Tabula Muris: transcriptomic characterization of 20 organs and tissues from Mus musculus at single cell resolution. https://www.ncbi.nlm.nih.gov/geo/query/acc.cgi?acc=GSE109774.

77. You Y. Data and Scripts from the Benchmarking UMI-based single cell RNA-sea preprocessing workflows analysis. https://github.com/YOU-k/preprocess_benchmark.

\section{Publisher's Note}

Springer Nature remains neutral with regard to jurisdictional claims in published maps and institutional affiliations.

- fast, convenient online submission

- thorough peer review by experienced researchers in your field

- rapid publication on acceptance

- support for research data, including large and complex data types

- gold Open Access which fosters wider collaboration and increased citations

- maximum visibility for your research: over $100 \mathrm{M}$ website views per year

At $\mathrm{BMC}$, research is always in progress.

Learn more biomedcentral.com/submissions 